\title{
Plasma Wave Observations with GEOTAIL Spacecraft
}

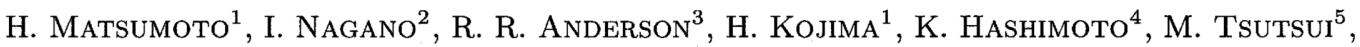 \\ T. OKADA ${ }^{6}$, I. KIMURA ${ }^{7}$, Y. OMURA ${ }^{1}$, and M. OKADA ${ }^{1}$ \\ ${ }^{1}$ Radio Atmospheric Science Center, Kyoto University, Uji, Kyoto 611, Japan \\ ${ }^{2}$ Department of Electrical and Computer Engineering, Kanazawa University, Kanazawa 920, Japan \\ ${ }^{3}$ Department of Physics and Astronomy, The University of Iowa, Iowa City, IA 52242, U.S.A. \\ ${ }^{4}$ Department of Electrical Engineering, Tokyo Denki University, Tokyo 101, Japan \\ ${ }^{5}$ Institute for Computer Sciences, Kyoto Sangyo University, Kyoto 603, Japan \\ ${ }^{6}$ Department of Electronics and Information, Toyama Prefectural University, Kosugi, Toyama 939-03, Japan \\ ${ }^{7}$ Department of Electrical Engineering II, Kyoto University, Kyoto 606, Japan
}

(Received October 6, 1993; Revised December 3, 1993; Accepted December 6, 1993)

The main scientific objectives of GEOTAIL Plasma Wave Instrument (PWI) are to investigate the characteristic features of wave phenomena which are generated by a variety of plasma processes occurring within the Earth's magnetosphere.

The PWI measures plasma waves in the frequency range from $5.62 \mathrm{~Hz}$ to $800 \mathrm{kHz}$ for the electric components and from $5.62 \mathrm{~Hz}$ to $12.5 \mathrm{kHz}$ for the magnetic components. The instrument is composed of three distinct sets of receivers: (1) the Sweep Frequency Analyzer (SFA), (2) the Multi-Channel Analyzer (MCA) and (3) the Wave-Form Capture (WFC). The first two receivers are dedicated to wave spectra measurement, while the last one is used to capture actual wave forms of two electric and three magnetic field components for the measured plasma wave emissions.

The present paper describes the PWI subsystems and their functions. We also report some results from initial observations made during the traversal of the geomagnetic tail and a skimming pass of the dayside magnetopause. These observational results are useful in providing a good overview of the PWI capabilities as well as elucidating the characteristic features of the wave spectra in these regions.

\section{Introduction}

Scarf et al. (1980) proposed to provide instrumentation to study the wave-particle interactions found near the Earth's magnetic equator in the original US OPEN program. A fundamental objective of the so-called OPEN program was to analyze the flow of mass, momentum and energy through the solar wind/magnetosphere/ionosphere system. Many of the physical processes controlling the entry, transport, storage, acceleration and loss of plasma in the Earth's magnetosphere are related directly to the underlying wave-particle interactions.

To meet the requirement for studying wave-particle interactions, design and manufacturing specifications for a Plasma Wave Instrument (PWI) on board the GEOTAIL spacecraft were solicited. In the design of the subsystem, an attempt in simultaneously capturing wave forms of two electric and three magnetic components of the waves was proposed, in addition to the highly sensitive measurement of electric and magnetic field spectra with fine frequency and time resolution.

During the period from October 1982 to September 1983, the ISEE-3 spacecraft performed the first scientific survey of the distant magnetic tail (Tsurutani and Rosenvinge, 1984). Based on the observational results of the ISEE-3 studies, several observational areas of interest to be emphasized by GEOTAIL were proposed by Scarf (1985) and other PWI members (Matsumoto, 1985; Nagano, 1985; Hashimoto, 1985; Omura, 1985). These proposals addressed those aspects 
of the wave phenomena which remained unclear and pointed out where more details investigation was warranted. The following paragraphs contain a brief overview of their main points.

1. Intense broadband electrostatic noise (BEN) has been observed not only in the near-Earth regions of the magnetosphere (Rodriguez and Gurnett, 1975; Anderson, 1984; Gurnett and Frank, 1977, 1978; Scarf et al., 1972; Gurnett et al., 1979; Catell et al., 1986) but also in the tail regions (Gurnett et al., 1976; Scarf et al., 1974, 1984a; Nishida et al., 1985). The ISEE-3 spacecraft observed BEN in the magnetosheath, the lobe and the lowlatitude boundary layer (Scarf et al., 1984a), as well as in conjunction with sheath flux ropes (Kennel et al., 1986) and slow shocks (Sibeck et al., 1984). Many theoretical studies directed towards understanding the generation mechanism of BEN have been published (Huba et al., 1978; Grabbe, 1987; Schriver and Ashour-Abdalla, 1987). Strong and persistent efforts by Schriver and Ashour-Abdalla $(1987,1989,1990)$ have been undertaken in an attempt to explain the BEN generation mechanism by computer experiments. Their work provides a synopsis of the current state of understanding for the generation of BEN in the plasma sheet boundary layer (PSBL), showing that it is generated by interactions between hot ion beams accelerated in the distant tail region and cold ions flowing from the polar ionosphere. However, their theory and simulation results cannot provide a sufficient explanation for the frequencies of the noise spectra which are actually observed. The BEN does not consist of continuous waves, but impulsive ones as shown by Anderson et al. (1982), Nishida et al. (1985), and Tsutsui et al. (1991). We should investigate further other possible generation mechanisms for BEN. The most useful step for solving this problem is to analyze the actual wave forms of BEN. Since the PWI has a wide band receiver for capturing wave forms, its analysis together with computer experiments would provide us with useful insight into the generation mechanism of BEN.

2. Auroral kilometric radiation (AKR) which was first observed by Gurnett (1974) and termed terrestrial kilometric radiation (TKR) in a relation to auroral substorms was examined using data from the IMP-6 spacecraft by Voots et al. (1977) and Kaiser and Alexander (1977). Subsequently, Slavin et al. (1984) used data from ISEE-3 to examine strong AKR correlated with the onset of substorms. The quantitative analysis of the correlation between strong AKR and turbulent phenomena in the distant tail region is important in clarifying direct electro-dynamic relations between the geomagnetic tail and the polar magnetosphere. The AKR generation mechanism in and around the localized polar magnetosphere have been treated both theoretically (Wu and Lee, 1979; Lee et al., 1980; Wu et al., 1982) and observationally ones (e.g., Ungstrup et al., 1990). If the GEOTAIL observations in the distant magnetic tail could establish a one-to-one correspondence between the AKR activity and plasma turbulence in the tail, this would add greater insight into the tail-polar ionospheric coupling and its role in AKR generation.

3. Scarf et al. (1984a) found relatively weak electromagnetic continuum radiation showing specific polarization patterns which indicated multiple sources and lower cutoff at the plasma frequency in the tail lobe. Coroniti et al. (1984) examined angular distributions of the continuum radiation intensities in the equatorial plane of the tail using the ISEE-3 data. The observation method developed by Gurnett et al. (1988) and a more detailed analysis of intensity as well as polarization of waves in the tail region provide us with important information on tail structure such as the opening of the tail magnetosphere.

4. Electron whistler-mode noise bursts were found by Scarf et al. (1984b) when ISEE-3 traversed the plasma sheet. Comparing them with those observed in other regions would provide useful information on their generation mechanism and its evolution under different plasma conditions.

5. Tsurutani and Smith $(1974,1977)$ carried out statistical studies of Chorus wave emissions from OGO-5 which has orbits with apogees of around 10 Re. In the former paper (Tsurutani 
and Smith, 1974), a relation between occurrence of Chorus emission and AE indices was confirmed. The orbit of the GEOTAIL spacecraft has a perigee of around $10 \mathrm{Re}$ on the dayside. Thus, Chorus emissions can be measured by GEOTAIL. Since the PWI has the capability to capture wave forms, the wave vectors, wave polarizations and Poynting flux of Chorus, Lion Roars and other electromagnetic mode waves can be estimated by analyzing the wave form data. Further, a detailed analysis of the Poynting vectors from whistler mode events observed in association with the $\mathrm{AE}$ indices could provide more information on details of their generation mechanism.

Many examples of plasma waves detected in conjunction with slow shock crossings near the magnetic reconnection region (Scarf et al., 1984b), tailward passage of plasmoids (Slavin et al., 1984) and magnetotail flux ropes have been reported. However, the basic plasma mechanisms responsible for the generation of these wave emissions remain unclear. Therefore, a quantitative classification of wave characteristics and behavior using the GEOTAIL data is one of the important tasks for providing a clear understandings of the electro-dynamic behavior of the magnetosphere.

The GEOTAIL spacecraft was launched from Kennedy Space Center at 14:26 UT on July 24, 1992. The primary scientific objectives of the PWI are to characterize wave-particle interaction processes and instabilities associated with the dynamics in the different regions of the magnetosphere with emphasis placed on examining in greater detail the phenomena described above. In the following section, we will describe the PWI subsystem and its various components in greater detail, followed by an overview of some of the initial wave observations.

\section{Description of the PWI Subsystems}

The PWI consists of three different sets of receivers: (1) the Sweep Frequency Analyzer (SFA), (2) the Multi-Channel Analyzer (MCA) and (3) the Wave-Form Capture (WFC). The first two sets of receivers are devoted to measuring wave spectra, while the last one is designed to capture wave forms from two electric and three magnetic field components of the measured wave emissions, simultaneously. The frequency ranges of the electric and the magnetic fields which can be measured range from $5.6 \mathrm{~Hz}$ to $800 \mathrm{kHz}$ and from $5.6 \mathrm{~Hz}$ to $12.5 \mathrm{kHz}$, respectively. The frequency range of wave forms which can be captured by the WFC is from $10 \mathrm{~Hz}$ to $4 \mathrm{kHz}$. The block diagram of the PWI system is shown in Fig. 1.

PWI observation data are available in three types of telemetry formats called Format 1, 2, and 3. Format 1 includes the SFA and MCA data. These data are recorded onboard magnetic data recorder and played back to NASA Deep Space Network.

Format 2 is a real time observation mode supported by Usuda Deep Space Center (UDSC) in Japan. It includes all of PWI observation data with the highest resolution.

Format 3 is an unusual observation mode. We mainly use this format, when we need to monitor US SIs' data in real time, because Format 2 does not include any US SIs' data. Format 3 includes all of PWI observation data, however, WFC data quaities are coarse relative to those in Format 2 .

\subsection{Configuration of PWI sensors}

In order to measure the weak electric and magnetic fields from plasma waves in the geomagnetic tail region, we have designed two kinds of electric sensors and tri-axial search coils, having taken into account both the most suitable condition for field detection and reduction of spacecraft noise. Figure 2 shows the configuration of the GEOTAIL spacecraft along with the definition of spacecraft coordinates and their relation to the antenna axes. Two sets of long dipole antennas with a length of $100 \mathrm{~m}$ tip-to-tip are dedicated to measuring the electric field. They are wire and probe antennas termed 'WANT' and 'PANT', respectively. 


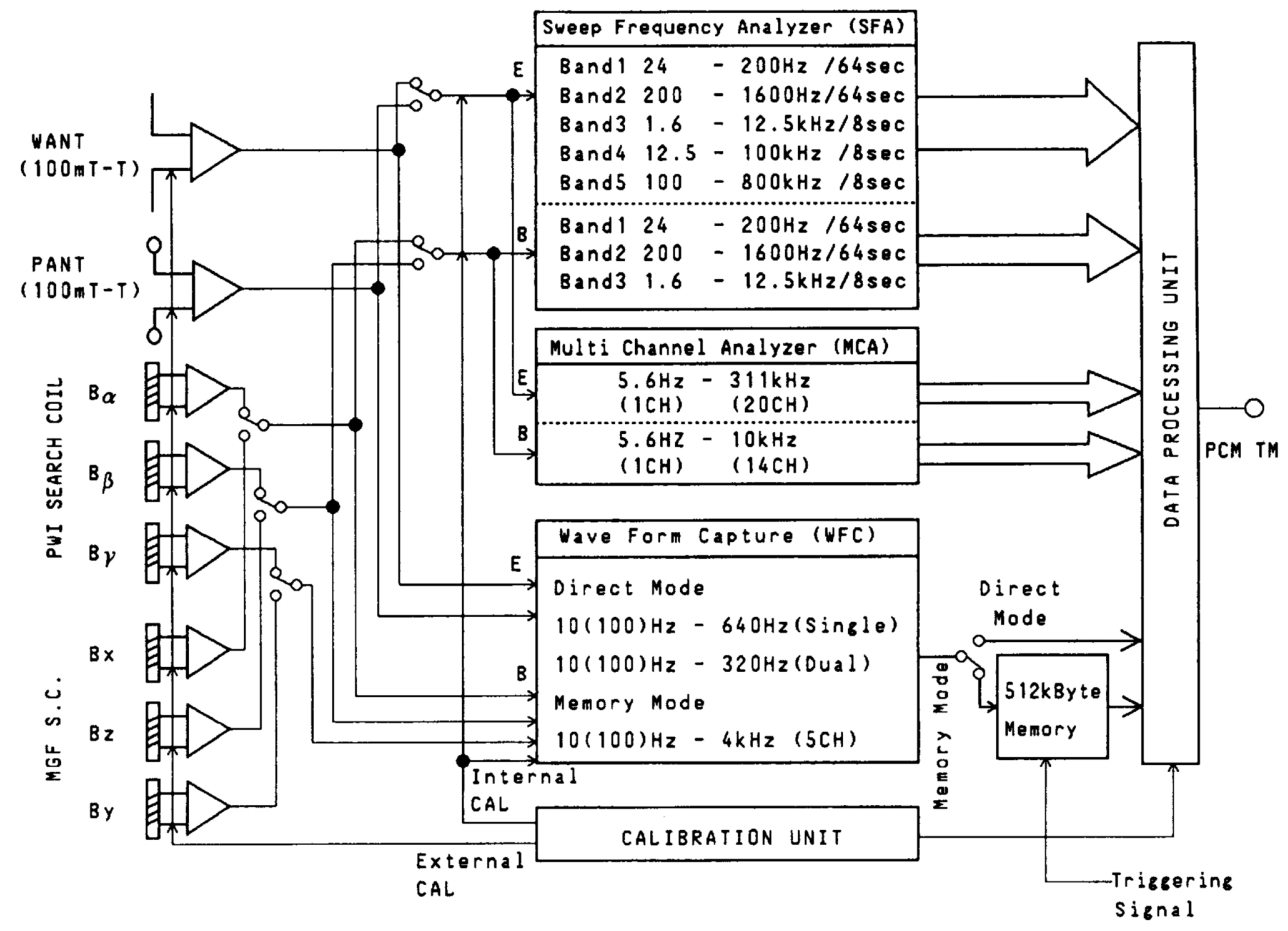

Fig. 1. Block diagram of the PWI system. The system is composed of three different functioning receivers: the Sweep Frequency Analyzer (SFA), Multi Channel Analyzer (MCA) and Wave Form Capture (WFC). Measurements of the electric fields are made with two dipole antennas, WANT and PANT, which are $100 \mathrm{~m}$ in length from tip-to-tip and are deployed orthogonally to each other. For measurements of the wave magnetic field, tri-axial search coils, $B_{\alpha}, B_{\beta}$ and $B_{\gamma}$, of the PWI are used. MGF search coils can be used if necessary.

Two masts of $6 \mathrm{~m}$ in length are used for mounting the magnetic field sensors well away from the spacecraft in an attempt to the reduce the spacecraft noise. One of these masts, called 'MSTF', mounts two sets of the flux-gate magnetometers which are used by the MGF team. They are dedicated primarily to the measurement of the DC magnetic field. Two sets of search coil and their pre-amplifiers which are used by both the PWI and MGF teams to measure wave magnetic fields or magnetic field fluctuations are mounted on the other mast called 'MST-S'.

In Fig. 2, the spacecraft coordinates $X$ and $Y$ are schematically shown by planar crosssections in order to show the orientations of the antennas more clearly. The antenna elements of the WANT and PANT are radially deployed from the spacecraft and orthogonal to each other. Their axes, $U$ and $V$, deviate by $15^{\circ}$ clockwise from the $X$ and $Y$ coordinate directions, respectively. The assembly of PWI tri-axial search coils (which is hereafter called PWI-SC) and their pre-amplifiers is mounted on the top of the MST-S. The tri-axial search coils associated with the MGF (MGF-SC) are mounted on the same mast but $2 \mathrm{~m}$ inside of the PWI-SC. The PWI-SC is normally connected to the PWI measurement system. However, we can select the MGF-SC by a telemetry command if necessary. The details on the MGF-SC are provided elsewhere in this issue (Kokubun et al., 1994). The direction of the MST-S (and MST-F) deviates by $45^{\circ}$ clockwise from the spacecraft $Y$ (and $-Y$ ) axis. The three axes of the PWI search coils are defined in a cylindrical coordinate system as follows: $\alpha$ is in the tangential direction, $\beta$ measures in the radial direction, and $\gamma$ is parallel to the spin $Z$ axis of the spacecraft. 


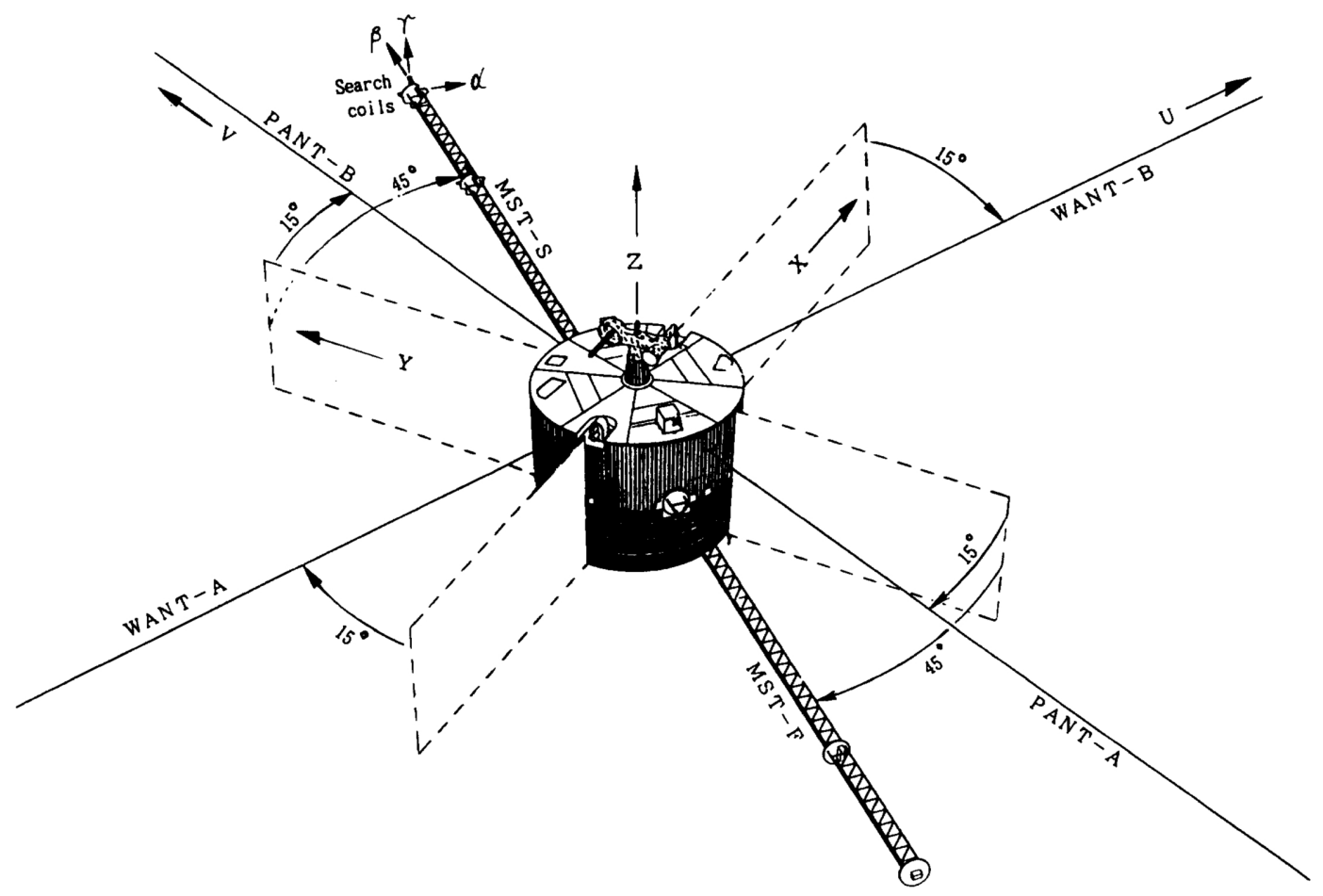

Fig. 2. Configuration of the PWI antennas deployed from the GEOTAIL spacecraft. Spacecraft coordinates are $\pm X$ and $\pm Y$ shown by four screens (broken lines) which does not actually exist but are drawn schematically in order to specify the orientations of antennas and masts. The WANT and PANT antenna elements are in the $\pm U$ and $\pm V$ directions, respectively, which deviate $15^{\circ}$ clockwise from the $X$ and $Y$ directions as viewed from the top of the spacecraft. The PWI tri-axial search coils are shown by $\alpha, \beta$ and $\gamma$ directions and are mount on the top of the MST-S which deviates $45^{\circ}$ from the $Y$ direction. The $\alpha$ and $\beta$ are the tangential and radial directions, respectively, in spacecraft cylindrical coordinates. Thus, $\gamma$ is parallel to the spacecraft spin axis, the $Z_{\text {gtl }}$ direction. The other search coils for the MGF are installed $2 \mathrm{~m}$ radially inward from those of the PWI.

\subsection{Electric field sensors}

The GEOTAIL spacecraft is equipped with the types of electric field measurement capabilities in the form of the PWI and EFD. The main purpose of the PWI is the detection of wave electric fields whereas the EFD measures DC electric fields in the magnetospheric plasma. Electric potentials picked up by the antennas are fed to both the PWI and EFD main electronics having already passed through their own individual pre-amplifiers. Both antennas, WANT and PANT, are basically electric dipoles. Pre-amplifiers for each antenna element are placed behind the antenna deploying mechanism which is mounted on top of the upper equipment shelf in the spacecraft.

\subsubsection{WANT}

The WANT is a wire dipole antenna. Figure 3(a) shows the outline drawing of the WANT antenna element. The element is a stainless steel wire of $50 \mathrm{~m}$ in length and $1.05 \mathrm{~mm}$ in diameter. The surface of the wire, except for portion of $10 \mathrm{~m}$ from the tip, is coated with a thin $(0.12 \mathrm{~mm})$ Polyamide film whose resistance coefficient is $10^{18} / \mathrm{cm}$ to insulate it electrically from the ambient plasma. The base of the wire from the antenna deploying mechanism out to $3 \mathrm{~m}$ is shielded by a copper-mesh sleeve in order to suppress the electrical interference radiated from the spacecraft. 


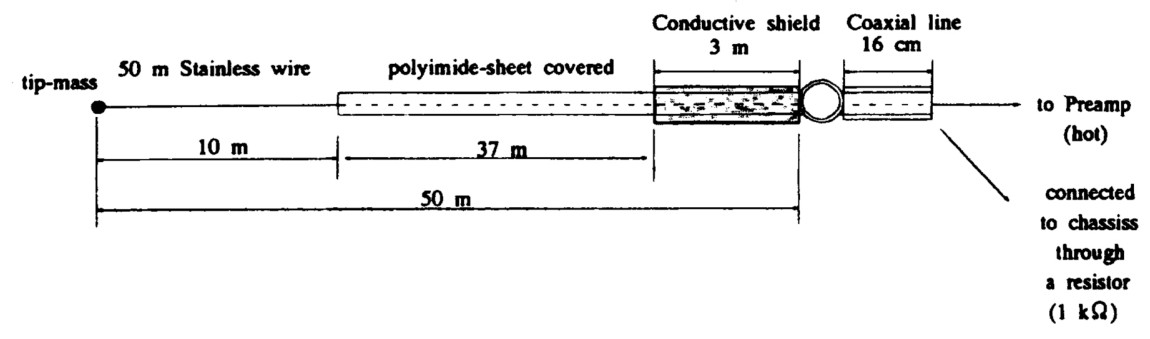

(a)

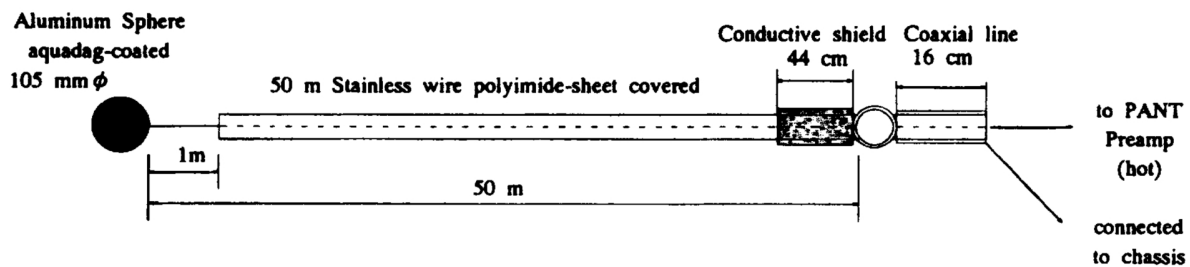

(b)

Fig. 3. Outline drawings of (a) WANT and (b) PANT elements. Electrical insulator (Polyamide sheet) coats each stainless steel wire for the sake of improving the DC electric field resolution. Near the spacecraft a conductive shield is used to suppress radiated noise from the spacecraft.

The copper-mesh sleeve is connected to the spacecraft chassis through a breeding resistance of $1 \mathrm{k} \Omega$.

The PWI makes use of the WANT in two different modes: one is a dipole mode and the other is a monopole mode. The instrument is nominally operated in the dipole mode. The purpose of the monopole mode is to detect spatial inhomogeneity in the plasma potentials around the spacecraft which might be formed by a plasma wake. The selection of the operation mode (dipole and monopole) is carried out by a telemetry command.

Figure 4(a) shows the electric circuit for the WANT and its pre-amplifier. This figure includes the equivalent sheath impedance around the antenna element and the stray and input capacitances formed between the input terminals of the pre-amplifier and the chassis. The electric potential induced on the WANT element is fed to the pre-amplifier input terminal through a decoupling capacitance of $220 \mathrm{pF}$. Since an input resistance of $200 \mathrm{M} \Omega$ is connected between the pre-amplifier and the chassis, the lower limit of the passing frequency becomes $3.6 \mathrm{~Hz}$.

In order to calibrate the amplitude and phase of the observed signal, a calibration signal from the PWI main circuit is imposed (CAL Mode) onto the WANT element through a resistance of $2 \mathrm{k} \Omega$ (the lower side of switch K2). Further, we can measure the antenna sheath impedance (IMP Mode) using the same calibration signal by switching the resistance to the higher value $5.1 \mathrm{M} \Omega$ (the upper side of K2). In this mode, the pre-amplifier picks up a different potential from that in the CAL mode because the potential is modified by the sheath impedance formed around the antenna elements.

\subsubsection{PANT}

The PANT is a pair of top-hat antennas composed of a sphere attached at each end of a stainless wire $50 \mathrm{~m}$ in length and $1.05 \mathrm{~mm}$ in diameter (same as WANT). Figure 3(b) shows an outline drawing of the PANT element. A hollow aluminum sphere of $105 \mathrm{~mm}$ in diameter is attached to the tip of the wire. The sphere surface is coated with Aquadag to ensure uniform 


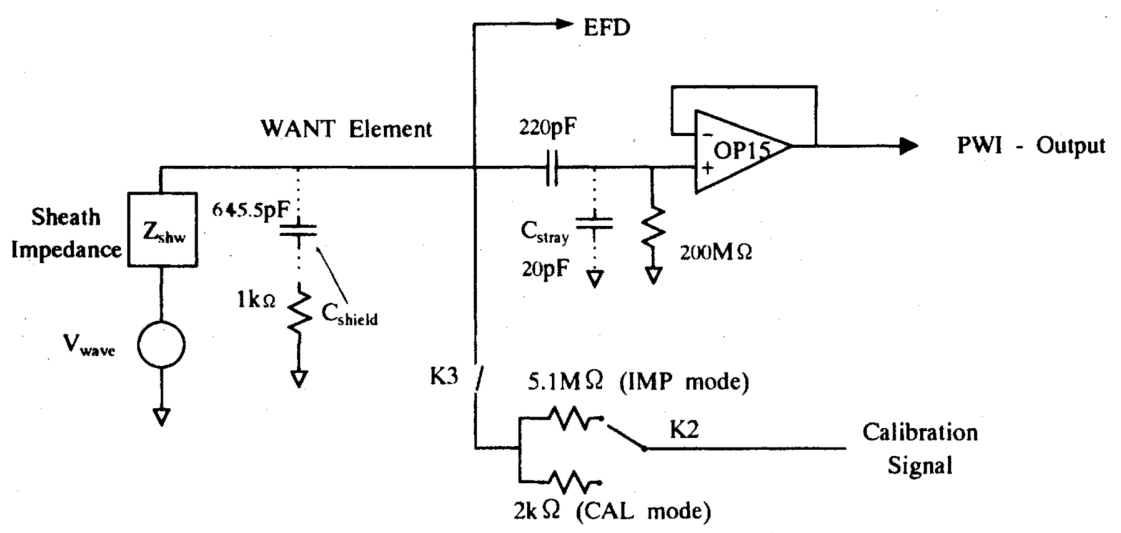

(a)

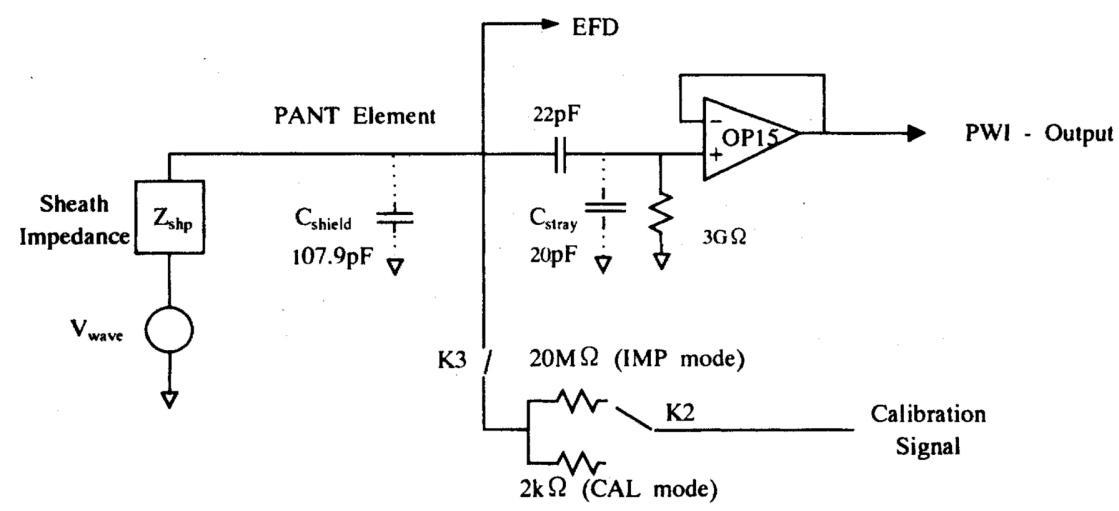

(b)

Fig. 4. Electric circuits for the antenna and pre-amplifiers of (a) WANT and (b) PANT. Equivalent values of sheath impedances $Z_{\text {shw }}$ and $Z_{\text {shp }}$ formed around the antenna elements are also depicted between a signal source $\left(V_{\text {wave }}\right)$ representing the plasma wave and the antenna element. $C_{\text {shield }}$ is the capacitance formed between the antenna element and its shield sleeve. $C_{\text {stray }}$ is the stray capacitance formed between the input terminal of the pre-amplifier and the chassis.

emission of photo-electrons. The entire wire surface except the outer portion $1 \mathrm{~m}$ from the sphere, is coated with Polyamide film. The inner portion extending $44 \mathrm{~cm}$ from the base of the wire is shielded with a copper-mesh sleeve connected directly to the chassis to suppress spacecraft noise.

Figure 4(b) shows the electric circuit for the PANT pre-amplifier. Because of the necessity for sensitive DC electric field measurements, the EFD team requires the input impedance of the pre-amplifier circuit to be larger than $5 \mathrm{G} \Omega$ at a frequency of $0.3 \mathrm{~Hz}$. Therefore, the decoupling capacitance and the input resistance connected to the PWI pre-amplifier were chosen to be $22 \mathrm{pF}$ and $3 \mathrm{G} \Omega$, respectively. This means that the lower limit of the passing frequency for this circuit is $2.4 \mathrm{~Hz}$. The measured stray and input capacitances at the input terminal of the pre-amplifier is $20 \mathrm{pF}$. By these two capacitances $(22 \mathrm{pF}$ and $20 \mathrm{pF}$ ), the signal amplitude picked up by the PANT antenna is reduced to about half level at the input terminal of the pre-amplifier. The total 
gain of the PANT circuit then becomes $-5.6 \mathrm{~dB}$ even though the gain of the pre-amplifier itself is unity.

The methods for calibration and the measurement of the antenna sheath impedance are the same as those of the WANT, though the resistance used for the IMP mode is $20 \mathrm{M} \Omega$ as shown in Fig. 4(b).

Figure 5 shows the minimum noise levels of the WANT and the PANT pre-amplifiers measured during the pre-flight calibration tests. The levels shown in the figure were obtained from the output of front-end circuits, when both input terminals of pre-amplifiers are connected to the ground. The frequency characteristics of the WANT noise level coincide with the theoretical value. The front-end circuit for WANT is shown in Fig. 4(a), whereas that for PANT includes an additional operational amplifier connected in cascade after the circuit shown in Fig. 4(b). Thus noise level of PANT is higher than that of WANT.

\subsection{Magnetic field sensors}

2.3.1 Tri-axial search coils

Two sets of tri-axial search coils, the PWI-SC and MGF-SC, are mounted on the MST-S mast. The PWI-SC measures wave magnetic fields over a frequency range up to $12 \mathrm{kHz}$, while the MGF-SC covers a frequency range up to $1 \mathrm{kHz}$. Each axial search coil of the PWI-SC is constructed from 20,000 turns of a copper wire $0.1 \mathrm{~mm}$ in diameter wound on a lightweight bobbin $30 \mathrm{~cm}$ in length. The coils are divided into 10 sectors over the central $20 \mathrm{~cm}$ portion of the bobbin to reduce the stray capacitance among the wound wire. At the center of the each main (search) coil, additional two sets of 2-turn coils are provided to form a negative flux feedback and for supplying the calibration signal. The core of these coils, which is situated inside the bobbin, consists of laminated layers $(0.05 \mathrm{~mm}$ thick) of parmalloy which has a high permeability. The layers are electrically insulated from each other in order to suppress the formation of eddy currents. The core is $30 \mathrm{~cm}$ long and has a cross-section of $5 \mathrm{~mm}$ square. The total specific

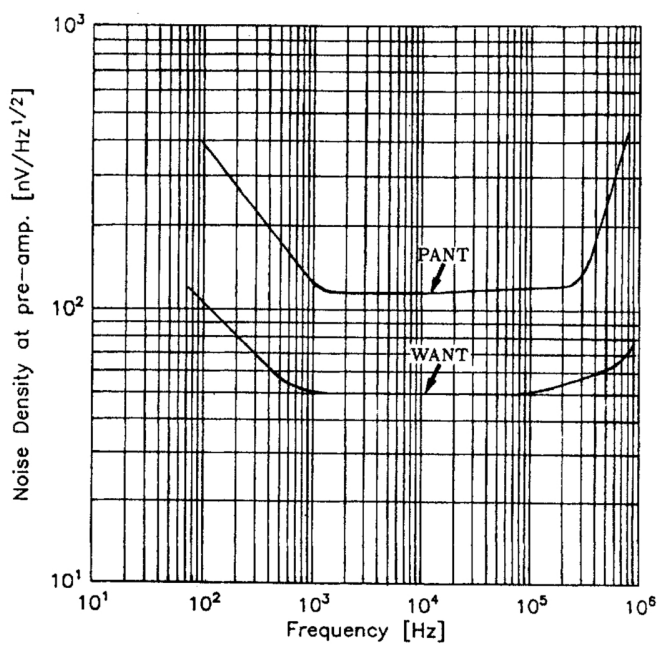

Fig. 5. Minimum noise levels of the WANT and PANT pre-amplifiers measured during the pre-flight calibration test. The levels shown in the figure were obtained from the output of front-end circuits, when both input terminals of pre-amplifiers are connected to the ground. The front-end circuit for WANT is shown in Fig. 4(a), whereas that for PANT includes an additional operational amplifier connected in cascade after the circuit shown in Fig. 4(b). Thus noise level of PANT is higher than that of WANT. 
permeability of the core is effectively 940 . Each main coil, together with the additional two sets of 2-turn coils, is covered with an aluminum ( $0.5 \mathrm{~mm}$ thick) housing having a dimension of $25 \mathrm{~mm} \times 25 \mathrm{~mm} \times 304 \mathrm{~mm}$. The housing having a slit along the coil axis serves as electrostatic shielding. The PWI-SC is assembled on an aluminum plate mounted on the top of the MST-S. The three search coils are deployed orthogonally to each other within a directional accuracy of $\pm 0.5^{\circ}$ after full extension of the MST-S. The aluminum plate induces some phase errors in our measurement of the null field direction with respect to the nearest search coil, which is about $3^{\circ}$ in the frequency range concerned. However, it turned out that this error does not affect the main direction of the field pattern which is used for determination of the wave properties such as the polarization phase.

\subsubsection{Pre-amplifier and its peripheral circuit}

Figure 6 shows a simplified illustration of a representative circuit diagram for the search coil pre-amplifiers. A negative flux feedback loop is introduced in order to suppress resonant characteristics of the search coil itself and to obtain a flat response in gain over the range of observation frequencies. The voltage induced in the main coil by the wave magnetic field is fed to the pre-amplifier with an open loop gain of $60 \mathrm{~dB}$. The output signal from the pre-amplifier drives the secondary coil through a divider with two resistances of $30 \mathrm{k} \Omega$ and $100 \Omega$, thereby magnetically coupling to the main coil and achieving the negative flux feedback.

Figures 7(a) and (b) show frequency response curves for the gain and phase rotation of a representative search coil ( $\gamma$ axis) and its pre-amplifier circuit when a magnetic field of 1 pT was applied to the coil axis. In (a), the characteristic curve shown corresponds to an applied magnetic field of $1 \mathrm{pT}$. This field strength was chosen to match the average order of magnitude field strength of waves expected in the geomagnetic tail, thereby showing the probable instrument performance

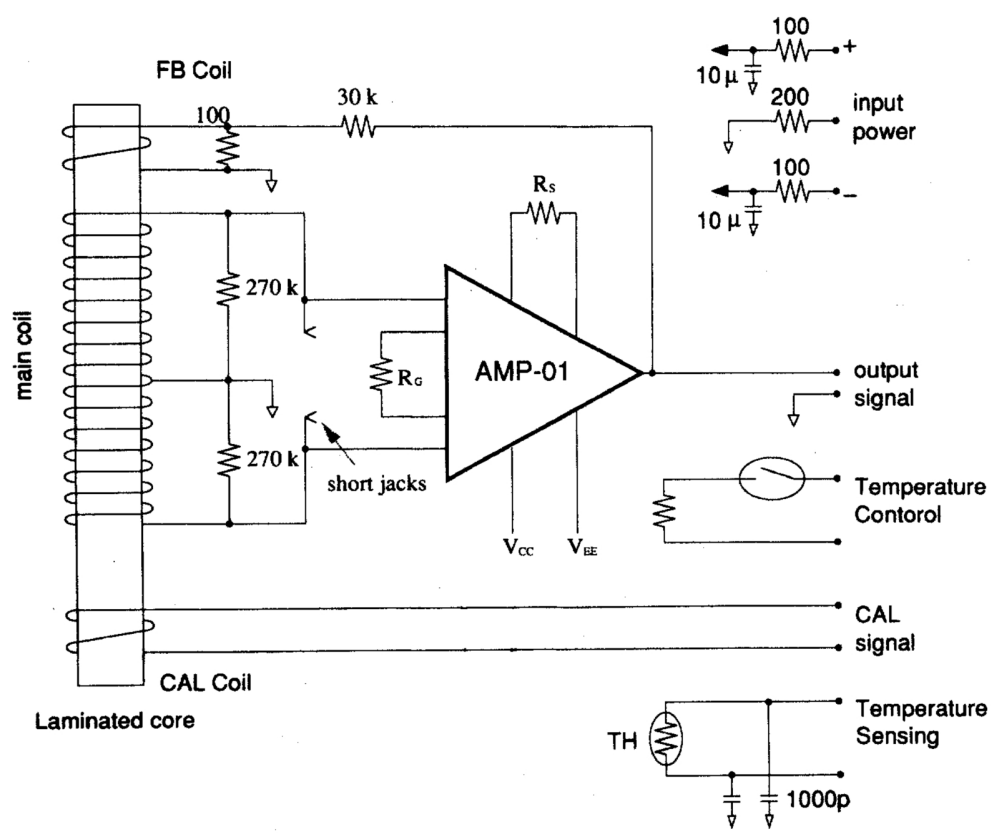

Fig. 6. Simplified illustration of the electric circuit diagram for the search coil and pre-amplifier. A negative feedback loop (FB) is introduced in order to suppress resonant characteristics of the search coil and to obtain a flat frequency response over the observation frequency range. A two-turn CAL coils is used for driving the calibration signal, which is connected in series to the other two search coil systems. 


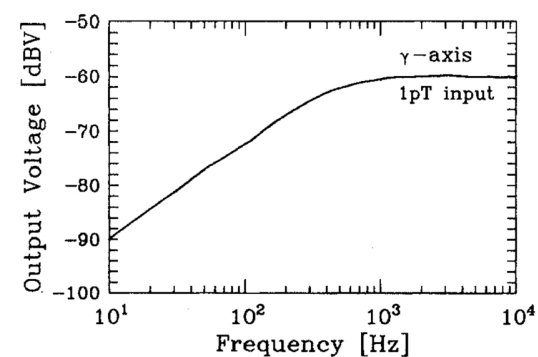

(a)

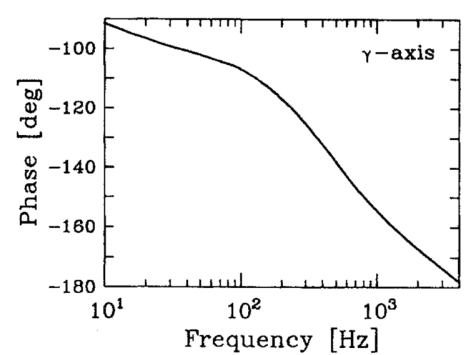

(b)

Fig. 7. (a) Frequency response of the gain for a representative search coil $(\gamma)$ and its pre-amplifier circuit. The measurement was conducted in a parallel magnetic field of $100 \mathrm{pT}$, but the characteristic curve illustrated in this figure represents the response which has been scaled down to the noise level ( $\sim 1 \mathrm{pT})$. (b) Phase rotation as a function of frequency for the same search coil and pre-amplifier circuit.

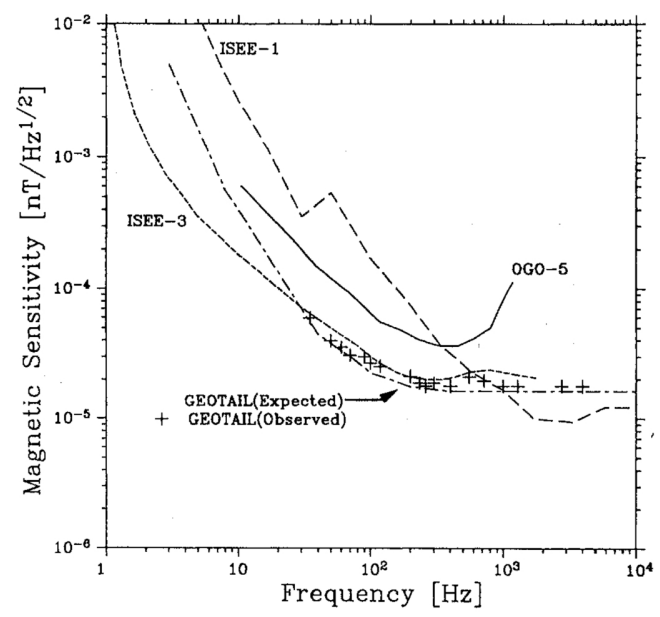

Fig. 8. Sensitivities of search coils on board several spacecraft measured in their flights. The dot-dashed curve shows theoretical values of the GEOTAIL PWI sensitivity. Search coil data of the other three spacecraft (Frandsen et al., 1969; Scarf et al., 1978; Gurnett et al., 1978) are noise levels observed in their flights. The + symbol indicates the minimum noise level of the GEOTAIL PWI measured in the geomagnetic tail region.

in this region. However, these results were deduced from the actual calibration measurements which were made using $100 \mathrm{pT}$ field strengths. The curve shows a linear increase in gain up to $400 \mathrm{~Hz}$, then constant for all frequencies above $400 \mathrm{~Hz}$. The responses for the two other axial search coils are nearly equal to the one depicted to within $\pm 1 \mathrm{~dB}$ for the gain and $\pm 3^{\circ}$ for the phase rotation.

Since the calibration coils for the three search coils are connected in series, a calibration signal with the same magnetic intensity is applied to each search coil. Therefore, the differences in the phases as well as the amplitudes among the three search coil circuits can be obtained precisely from the captured wave forms.

The measured in-flight sensitivities of the search coils as a function of frequency are plotted in Fig. 8. The dot-dash curve shows the expected theoretical sensitivity of the GEOTAIL PWI search coils. The + symbols indicate noise levels actually measured when the GEOTAIL spacecraft was 
in the geomagnetic tail region, and these clearly coincide well with the theoretical curve. The other curves in the figure represent the noise levels from the search coils on board ISEE-1 and -3 and OGO-5 (Scarf et al., 1978; Gurnett et al., 1978; Frandsen et al., 1969). This comparison shows clearly that the GEOTAIL PWI search coils have the highest sensitivity among this type of magnetic sensors for the frequency range from $30 \mathrm{~Hz}$ to $1 \mathrm{kHz}$.

A distance dependence in the noise intensity of $r^{-2.5}$ for the magnetic field was confirmed during the ground ElectroMagnetic Compatibility (EMC) test of the GEOTAIL spacecraft, where $r$ is the distance from the center of the spacecraft (Tsutsui et al., 1992). In-flight observations have demonstrated that mounting of the PWI-SC at $6 \mathrm{~m}$ in distance from the spacecraft surface has made a favorable reduction in the noise level measured by this instrument.

\subsection{Main electronics}

\subsubsection{Sweep Frequency Analyzer (SFA)}

The SFA provides spectral information on plasma wave amplitudes over the frequency range from $24 \mathrm{~Hz}$ to $800 \mathrm{kHz}$ for the electric field and $24 \mathrm{~Hz}$ to $12.5 \mathrm{kHz}$ for the magnetic field. The SFA consists of eight independent receivers covering 5 frequency bands for the electric fields and three frequency bands for the magnetic fields. The receiver specifications are listed in Table 1.

Figure 9 shows a block diagram of the main electronics of one of the eight receivers in the SFA which measures a single frequency band. The lowest frequency band (Band 1) is a single conversion super heterodyne receiver while the other frequency bands correspond to double conversion super heterodyne ones. Each receiver has a very good frequency resolution of $1 / 128$ of the receiver frequency band although their time resolution $(64 \mathrm{sec}$ for Bands 1 and 2, and $8 \mathrm{sec}$ for Bands $3 \sim 5$ ) is somewhat coarse. The "H/L" in Fig. 9 represents a gain control with a $30 \mathrm{~dB}$ difference and can be varied by a telemetry command. Frequencies of the LPF, the 1st and 2nd Local OSCs and the 1st and 2nd IFs in each band are listed in Table 2. The signal from the 2nd

Table 1. Specification of SFA.

\begin{tabular}{|c|c|r|r|l|r|}
\hline Band & Frequency Range & Freq. Step & Bandwidth & Source & Sweep \\
\hline 1 & $24 \mathrm{~Hz} \sim 200 \mathrm{~Hz}$ & $1.3 \mathrm{~Hz}$ & $2.6 \mathrm{~Hz}$ & $B$ and $E$ & $64 \mathrm{sec}$ \\
\hline 2 & $200 \mathrm{~Hz} \sim 1600 \mathrm{~Hz}$ & $10.7 \mathrm{~Hz}$ & $10 \mathrm{~Hz}$ & $B$ and $E$ & $64 \mathrm{sec}$ \\
\hline 3 & $1.6 \mathrm{kHz} \sim 12.5 \mathrm{kHz}$ & $85.4 \mathrm{~Hz}$ & $85 \mathrm{~Hz}$ & $B$ and $E$ & $8 \mathrm{sec}$ \\
\hline 4 & $12.5 \mathrm{kHz} \sim 100 \mathrm{kHz}$ & $683 \mathrm{~Hz}$ & $680 \mathrm{~Hz}$ & $E$ only & $8 \mathrm{sec}$ \\
\hline 5 & $100 \mathrm{kHz} \sim 800 \mathrm{kHz}$ & $5.47 \mathrm{kHz}$ & $5.4 \mathrm{kHz}$ & $E$ only & $8 \mathrm{sec}$ \\
\hline
\end{tabular}

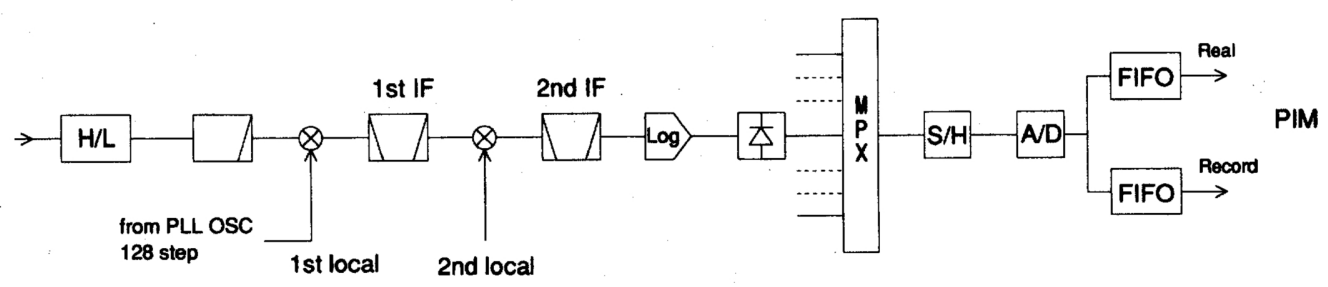

Fig. 9. Block diagram of the main electronics for a representative frequency band among the eight receivers in the SFA. Frequency resolution in the band is determined by 128 steps in the 1st local OSC. The sweep period of the 128 steps is $64 \mathrm{sec}$ for Bands 1 and 2, and $8 \mathrm{sec}$ for Bands 3,4 and 5 , respectively. This decides the time resolution of the observations. Quasi-logarithmic Amplifier "Log" has a dynamic range of $90 \mathrm{~dB}$. H/L is a gain controller with $30 \mathrm{~dB}$ switched by a telemetry command. 
Table 2. Frequency table of SFA.

\begin{tabular}{|c|r|c|r|r|r|}
\hline Band & LPF & 1st local & 1st IF & 2nd local & 2nd IF \\
\hline 1 & $250 \mathrm{~Hz}$ & $415.2 \sim 584.8 \mathrm{~Hz}$ & $390.6 \mathrm{~Hz}$ & - & - \\
\hline 2 & $1.875 \mathrm{kHz}$ & $3.322 \sim 4.678 \mathrm{kHz}$ & $3.125 \mathrm{kHz}$ & $2.84 \mathrm{kHz}$ & $289 \mathrm{~Hz}$ \\
\hline 3 & $12.8 \mathrm{kHz}$ & $26.57 \sim 37.43 \mathrm{kHz}$ & $25 \mathrm{kHz}$ & $20 \mathrm{kHz}$ & $5 \mathrm{kHz}$ \\
\hline 4 & $110 \mathrm{kHz}$ & $212.6 \mathrm{kHz} \sim 299.4 \mathrm{kHz}$ & $200 \mathrm{kHz}$ & $210 \mathrm{kHz}$ & $10 \mathrm{kHz}$ \\
\hline 5 & $880 \mathrm{kHz}$ & $1.701 \mathrm{MHz} \sim 2.395 \mathrm{MHz}$ & $1.6 \mathrm{MHz}$ & $1.61 \mathrm{MHz}$ & $10 \mathrm{kHz}$ \\
\hline
\end{tabular}

(a)

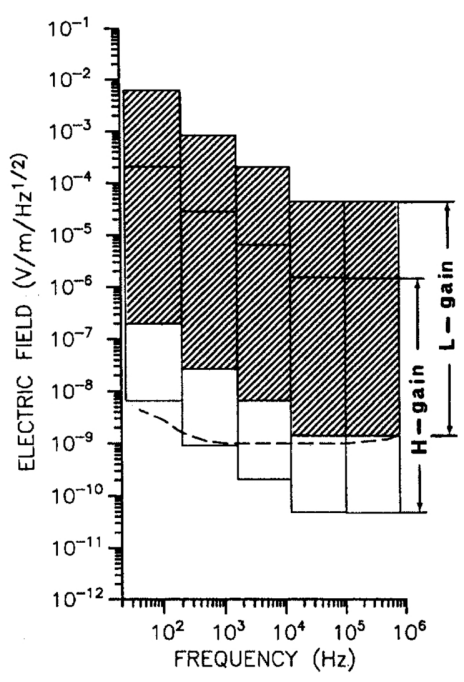

(b)

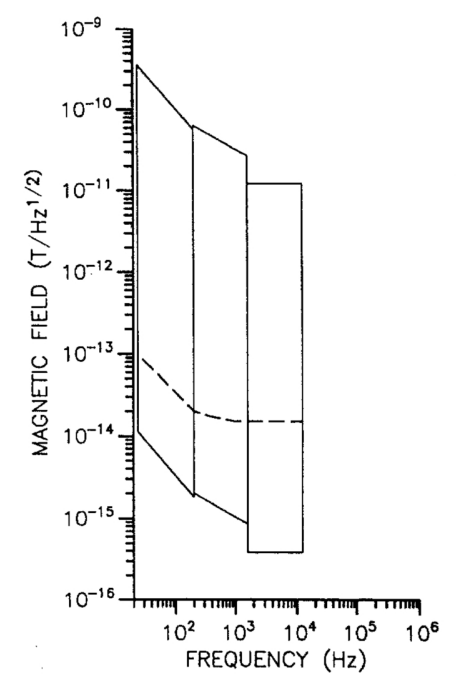

Fig. 10. SFA amplitude dynamic ranges of (a) five band receivers for the electric field, and of (b) three for the magnetic field. In each band of the electric field receivers, high $(\mathrm{H})$ and low $(\mathrm{L})$ gain amplifications with a 30 $\mathrm{dB}$ difference are selectable in keeping an inherent dynamic range $(90 \mathrm{~dB})$ of the circuit, while the sensitivity for magnetic field is constant. In each figure, a representative sensor sensitivity is drawn by a broken line.

IF amplifier passes through quasi-logarithmic amplifiers labeled "Log" where its signal amplitude is detected linearly then converted to digital data. In order to meet the requirements for total power consumption, low power ICs are used. This affected the final frequencies at the linear detector which were chosen to be rather low (see Table 2) in order to make use of operational amplifiers which could work with low power in such lower frequency ranges.

The SFA measures one wave electric field component of either the $E_{U}$ or $E_{V}$ component and one wave magnetic field component of either the $B_{\alpha}$ or $B_{\gamma}$ component. The selection of which field component is measured is carried out by a telemetry command. The dynamic range of the observed field intensities in each frequency band are shown in Fig. 10(a) for the electric field and (b) for the magnetic field. Each receiver has an effective dynamic range of $\sim 90 \mathrm{~dB}$. The electric field receivers are placed in the High gain mode by stepping up the level in the Low gain mode by $30 \mathrm{~dB}$. These two dynamic ranges are depicted in Fig. 10(a).

The SFA has another mode of operation which can be used. Despite its operation as the SFA, we can also fix the measured frequency to one preset value in each frequency band. This mode provides very high time resolution for the observed signals at an arbitrarily selected frequency. The time resolutions in this mode are $0.5 \mathrm{sec}$ for Bands 1 and 2, and $62.5 \mathrm{msec}$ for Bands 3, 4, 
and 5 , respectively. This mode change is also carried out by a telemetry command.

\subsubsection{Multi Channel Analyzer (MCA)}

This subsystem is provided by the University of Iowa. The MCA contains two spectrum analyzers with fixed frequency channel filters as shown in Fig. 11. It provides high time resolu-

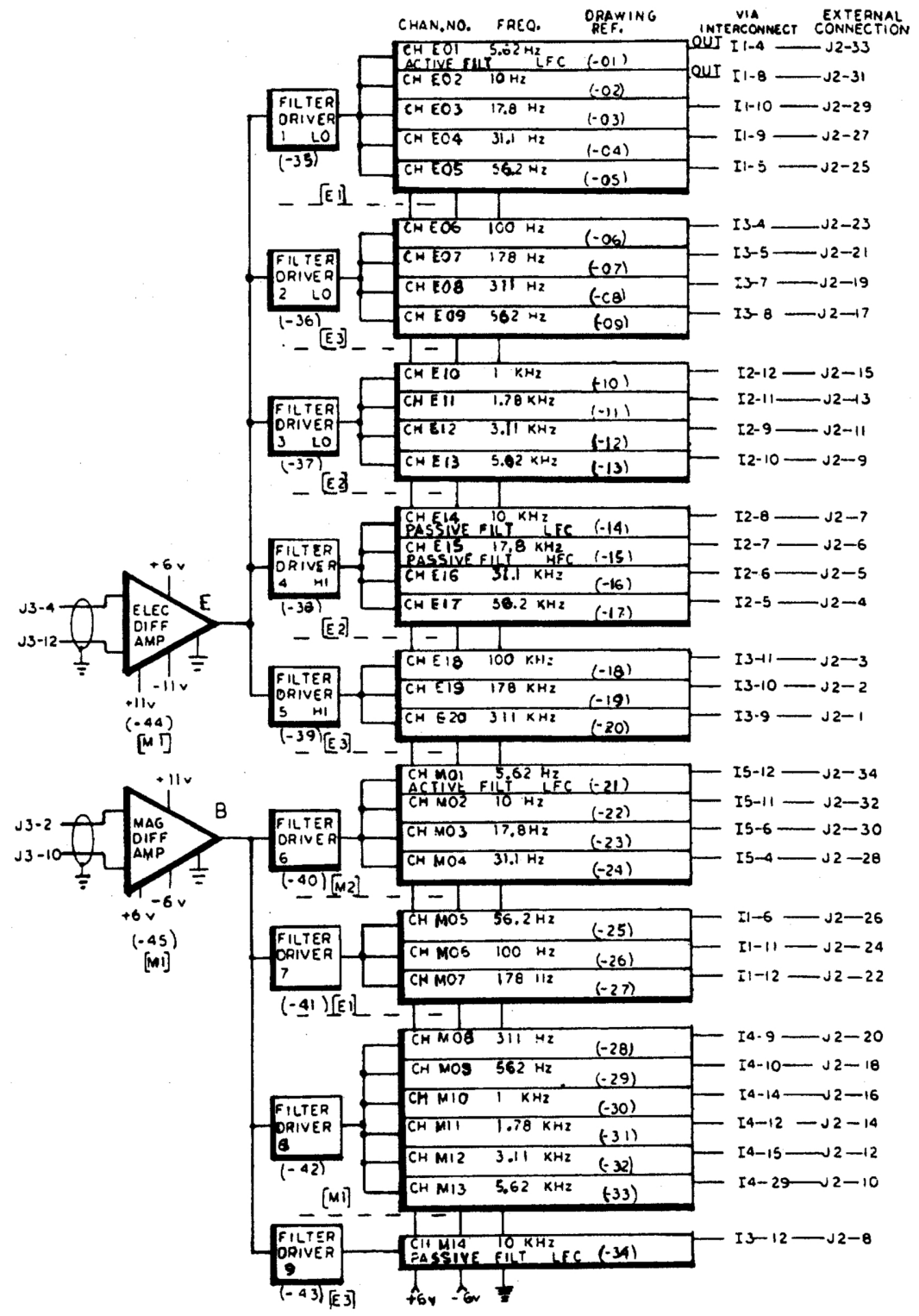

Fig. 11. Block diagram of the MCA system. The system contains two spectrum analyzers with fixed frequency channel filters. One of the analyzers is used for measure the electric field and is composed of 20 channels covering the frequency range from $5.62 \mathrm{~Hz}$ to $311 \mathrm{kHz}$. The other spectrum analyzer is used for magnetic field measurements and has 14 channels covering the frequency range from $5.62 \mathrm{~Hz}$ to $10 \mathrm{kHz}$. The bandwidths of the filters are $\pm 15 \%$ of the channel center frequency in the frequency range below $10 \mathrm{kHz}$ and $\pm 7.5 \%$ of the center frequency for frequency above $10 \mathrm{kHz}$. 
tion data to complement the coarser time resolution data of the SFA. However, their frequency resolution is coarse because they have only four frequency channels per decade in frequency.

The input signal to the MCA is provided from the output of the common front-end circuit of the PWI system as well as to SFA and WFC receivers. One multi-channel spectrum analyzer is used to measure the electric field and is composed of 20 channels covering the frequency range from $5.62 \mathrm{~Hz}$ to $311 \mathrm{kHz}$. The other spectrum analyzer is used for magnetic field measurements and has 14 channels covering the frequency range from $5.62 \mathrm{~Hz}$ to $10 \mathrm{kHz}$. The bandwidths of the filters are $\pm 15 \%$ of the channel center frequency in the frequency range below $10 \mathrm{kHz}$ and $\pm 7.5 \%$ of the center frequency for frequency above $10 \mathrm{kHz}$. The MCA instrument measures the wave electric field of either the $E_{U}$ or $E_{V}$ component (depending on which antenna is used) with a dynamic range of $\sim 110 \mathrm{~dB}$ and the wave magnetic field of either the $B_{\alpha}$ or $B_{\gamma}$ component with a dynamic range of $\sim 100 \mathrm{~dB}$.

Signals from all channels are sampled simultaneously so that the ratio of the electric to magnetic field strength may be calculated accurately. The signals are sampled once (data acquisition time is $1.037 \mathrm{msec}$ ) every $250 \mathrm{msec}$ in Format 2 (and every $500 \mathrm{msec}$ in Format 1). Each channel of the spectrum analyzer has a suitable integration time in the linear detection of signal ranging from $500 \mathrm{msec}$ in the lower frequency channels and decreasing to a few milliseconds for those channel above $3 \mathrm{kHz}$.

\subsubsection{Wave-Form Capture (WFC)}

The WFC data are used for the detailed analysis of the wave characteristics, such as determination of the wave vector, polarization, Poynting flux, and antenna sheath impedance. Figure 12 shows a block diagram of the WFC system. The system has five (two electric and three magnetic) receivers each connected to a sensor through each front end circuit. The WFC has two different operation modes: Memory mode and Direct mode.

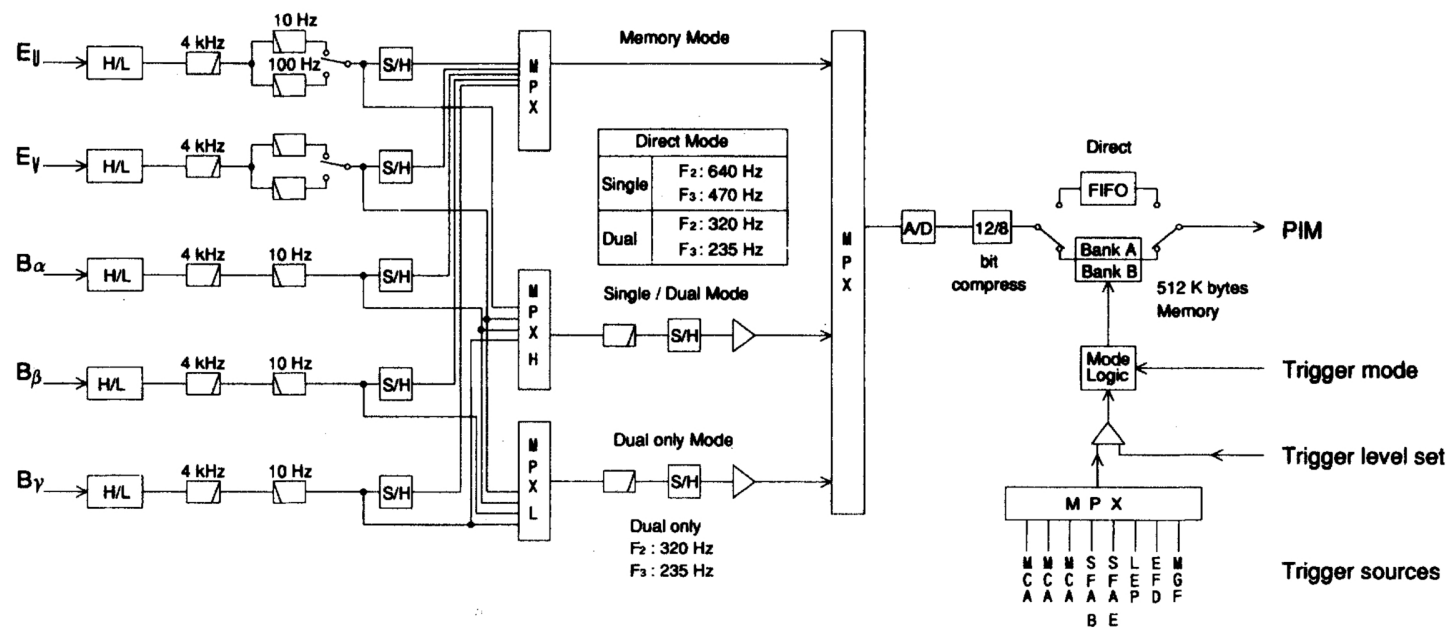

Fig. 12. Block diagram of the WFC system. The system has five receivers connected to five sensors. The dynamic range of each receiver is $66 \mathrm{~dB}$. Sensitivity of the receivers is controlled by "H/L" giving a $30 \mathrm{~dB}$ change. Additional HPFs with a cutoff frequency of $100 \mathrm{~Hz}$ are included in the circuits for the electric field and are selected when the noise interference from the spacecraft become strong in the low frequency range. In Memory Mode, A-D converted data of the wave forms from five field components (3 electric and 2 magnetic fields) are stored into the Memory Banks A and B. Triggering for the data storage into the memories is controlled by eight trigger sources shown in the figure in addition to a free running timing pulse (not shown in this figure). In Direct Mode, wave form(s) of one or two field components are telemetered directory to the ground, which are called Single or Dual Mode, respectively. 
In the Memory mode, wave signals are measured simultaneously as $E_{U} E_{V}, B_{\alpha}, B_{\beta}$, and $B_{\gamma}$. These are fed to a gain-controller followed by an anti-aliasing filter (LPF) with an upper frequency cutoff of $4 \mathrm{kHz}$, then through high pass filters (HPF) which have a low frequency cutoff of $10 \mathrm{~Hz}$. In Bands 1 and 2, an alternate HPF with a low frequency cutoff of $100 \mathrm{~Hz}$ can be used with the electric field measurements. Wave forms of the analog signal are sampled then converted into 12 bit data by an A/D converter with a frequency of $12 \mathrm{kHz}$. The 12 bit digital data are compressed into 8 bit by a quasi-logarithmic compression method. The compressed 8 bit data are then stored into onboard memory with a storage of $512 \mathrm{kBytes}$ for a period of $8.7 \mathrm{sec}$. The stored wave form data are read out of memory and telemetered to the ground using the PCM telemetry during a uninterrupted period of 275 seconds in telemetry Format 2 and 375 seconds in telemetry Format 3. The start timing for initiating wave form capture is controlled by either free running timing pulse (INTER. mode) or when the instrument receives one of eight different sources for triggering signals. The instrument is triggered when one of the following measurements exceeds a preset level:

1. The intensity of $Z$ axis component of the DC magnetic field as measured by the outboard fluxgate magnetometer of the MGF.

2. The amplitude of the plasma turbulence detected by a single probe of the EFD.

3. The magnitude of ion and/or electron moments as measured by the electrostatic analyzers of the LEP-EA.

4. The amplitude of the electric field measured on the $100 \mathrm{kHz}$ channel of the MCA.

5. The amplitude of the electric field measured on the $316 \mathrm{~Hz}$ channel of the MCA.

6. The amplitude of the magnetic field measured on the $10 \mathrm{~Hz}$ channel of the MCA.

7. The integral amplitude of the electric field over the entire frequency range of Band 1 (frequencies less than $250 \mathrm{~Hz}$ ) of the SFA.

8. The integral amplitude of the magnetic field over the entire frequency range of both Bands 1 and 2 (frequency less than $1.875 \mathrm{kHz}$ ) of the SFA.

The selection among the possible eight triggering sources and its preset level or amplitude is made via telemetry commands.

When the WFC instrument is operated in the Memory mode the following three modes of operation are available for storing and reading the data:

1. AFT: Storage of $512 \mathrm{kByte}$ of data after instrument triggering.

2. MID: Storage of two successive $256 \mathrm{kByte}$ sequences of data, one before and the other after instrument triggering.

3. BEF: Storage of $512 \mathrm{kByte}$ of data before instrument triggering.

In the last two modes, a continuous sequence of signal sampling and storage into the memory is repeated until a triggering signal is detected by the instrument.

A common problem of noise interference in the plasma wave instruments on spinning occurs as each group of solar cell circuits cycles on/off when the solar cell surface of the group faces to the sun-lit side then to the shadow side of the spacecraft. This repetition of the circuit from on to off to on again causes radiation of electromagnetic noises. To mitigate this problem, a high pass filter (HPF) with a cutoff frequency of $10 \mathrm{~Hz}$ is inserted in the receivers in order to suppress the detection of low frequency noise from the solar cell circuits. For the electric field receivers, we prepared an option for changing the HPF cutoff frequency from $10 \mathrm{~Hz}$ to $100 \mathrm{~Hz}$ in case the noise extended to higher frequencies then expected. The selection of the lower frequency cutoff is based upon the observed insitu noise level. These noise suppression measures limit frequency range of the observed wave forms to a band from $10 \mathrm{~Hz}$ (or $100 \mathrm{~Hz}$ ) to $4 \mathrm{kHz}$.

Besides the Memory Mode, the WFC can be operated in a Direct mode, with two possible variations: one is the Single channel mode in which only one field component is measured continuously and telemetered to ground on a real-time basis. In this operation mode the instrument can measure continuous wave forms of one electric or one magnetic component with an upper 
limit in frequency of $640 \mathrm{kHz}$ in telemetry Format 2 (or $470 \mathrm{~Hz}$ in telemetry Format 3). The other method of instrument operation is in the Dual channel mode where two field components are measured and telemetered alternately. In this mode the upper limit in frequency is $320 \mathrm{~Hz}$ in telemetry Format 2 (or $235 \mathrm{~Hz}$ in telemetry Format 3) for each component. For both of these mode, the data sampling frequency is three times the upper limit in frequency.

The WFC system can measure the wave amplitude with a dynamic range of $66 \mathrm{~dB}$. The receiver gain keeping its dynamic range can be stepped up in the gain controller " $\mathrm{H} / \mathrm{L}$ " by $40 \mathrm{~dB}$ and $20 \mathrm{~dB}$ in electric and magnetic channels to amplify the weak signal, respectively.

\subsubsection{Onboard calibrations and measurements of antenna sheath impedance}

In order to obtain absolute values of the observed wave amplitude and the phase differences between the different field components, a signal calibration system is included in the PWI system. We use two types of calibration signals; one is a sinusoidal wave form of $100 \mathrm{~Hz}$ and the other is a composite signal of six rectangular wave forms. Since the SFA has a wide frequency range extending up to $800 \mathrm{kHz}$, the frequency components of the composite signal are designed to encompass this entire frequency range (all five bands of the SFA receivers). The fundamental frequencies of the six rectangular wave forms are $10 \mathrm{~Hz}, 100 \mathrm{~Hz}, 1 \mathrm{kHz}, 10 \mathrm{kHz}, 100 \mathrm{kHz}$, and $1 \mathrm{MHz}$. For more precise calibration in the same frequency domain, they can be switched to their half frequency values by a telemetry command.

The calibration signal is supplied to all five sensors (both sets of dipole antennas and the triaxial search coils) simultaneously. The calibration operation is initiated via a telemetry command. The start time of the calibration is synchronized with the timing of the frequency sweep in the SFA measurements and the time duration of the calibration signal is the same as one sweep period. The amplitude attenuation of the calibration signal can be changed from 0 to $60 \mathrm{~dB}$ in $20 \mathrm{~dB}$ steps by telemetry commands. Examples of the frequency spectra for the calibration signal in the five electric field frequency bands and the three magnetic field frequency bands which compose the SFA are shown in Fig. 13.

For the analysis of SFA and MCA data, only the amplitude calibration is needed. However, for the WFC data, extremely precise measurements of both amplitudes and phase differences among the different field components are required. This is because the main objective in the analysis of the WFC data is to determine the wavevector, wave polarization and Poynting flux of the observed waves as well as to estimate the sheath impedance formed around the WANT and PANT antennas. The wave form data stored in the memory must be restored to that of each component $\left(E_{U}, E_{V}, B_{\alpha}, B_{\beta}\right.$ and $B_{\gamma}$ ) as measured at the respective sensor points using the appropriate transfer function. Although the transfer function obtained from pre-flight calibration data for each receiver circuit can be utilized for this purpose, there is a possibility that the instrument properties and hence the transfer function can change over times. Any temporal change in the transfer function will cause an erroneous estimation in the wave properties if used to restore the data. Thus, in case of possible changes in the pre-flight transfer functions, in-situ calibrations are indispensable for the reliability of the data analysis. An example of wave forms before and after the restoration using the pre-flight transfer function of the receiver circuit is shown in Fig. 14. The procedure used in restoring the wave forms is as follows: The telemetered wave form data is first transformed to frequency spectrum data using an FFT. The phase and amplitude in each frequency component are restored to those at the sensor point using the transfer function of the receiver circuit. The restored frequency spectrum is then transformed to the wave forms using an inverse FFT. The example in Fig. 14 shows the wave forms of a calibration signal imposed on a search coil ( $\mathrm{B}_{\beta}$ axis) on October 13, 1992. Figure 14(a) shows a wave form generated from the WFC telemetry data prior to applying the transfer function. The wave form data contain less low frequency components because the transfer function of the search coil circuit shown in Fig. 7 includes a high pass filter which remove these components. Figure 14(b) shows the wave 

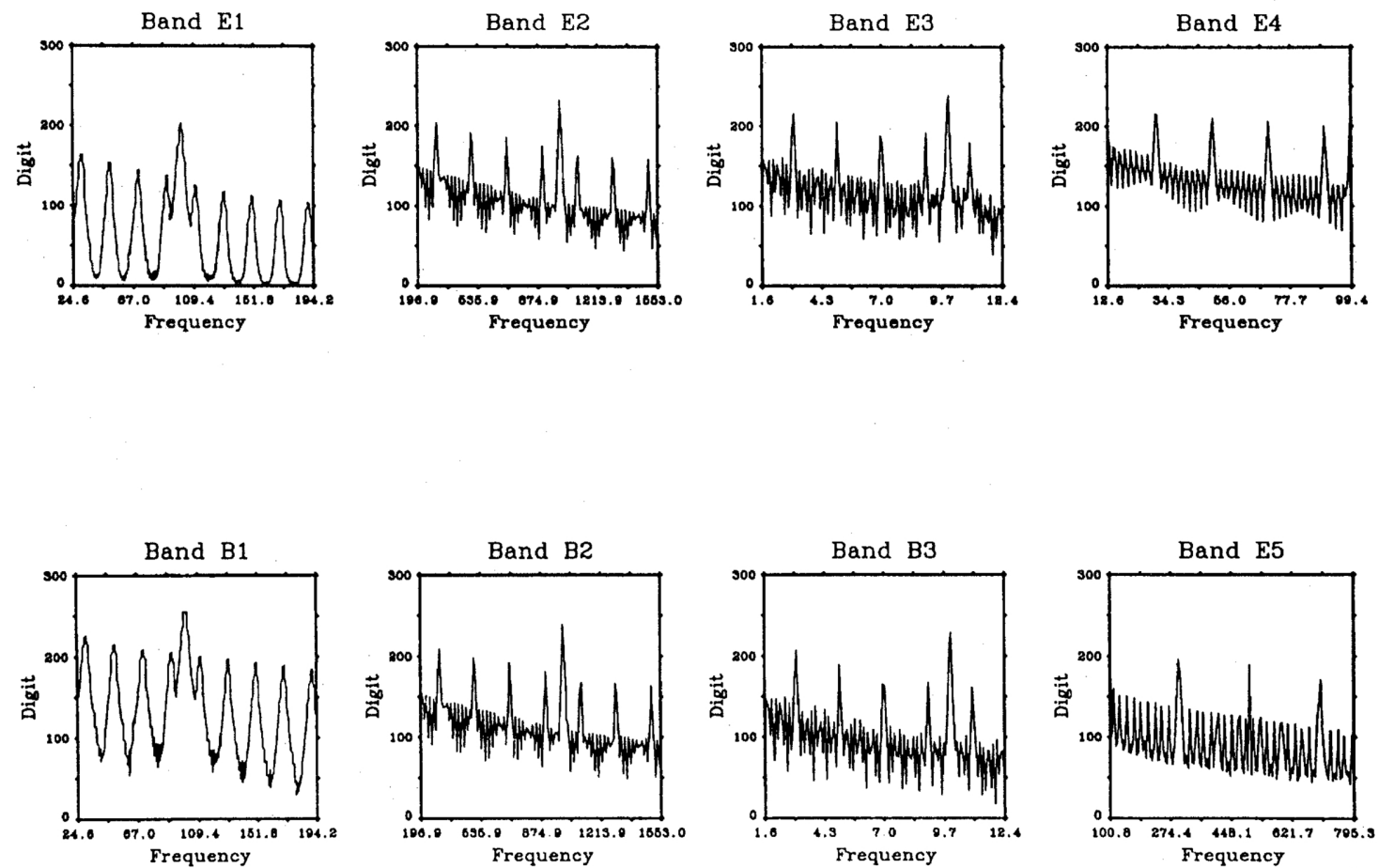

Fig. 13. SFA frequency spectra of the calibration signal shown in five-frequency band receivers for the electric field and three for the magnetic field. The calibration signal is composed of six rectangular wave forms with their fundamental frequencies at $10 \mathrm{~Hz}, 100 \mathrm{~Hz}, 1 \mathrm{kHz}, 10 \mathrm{kHz}, 100 \mathrm{kHz}$, and $1 \mathrm{MHz}$. In this display, the receivers are in Low gain mode, and the attenuation of the calibration signal is $0 \mathrm{~dB}$, though its amplitude can be attenuated to $-60 \mathrm{~dB}$ by stepping every $20 \mathrm{~dB}$.

(a)

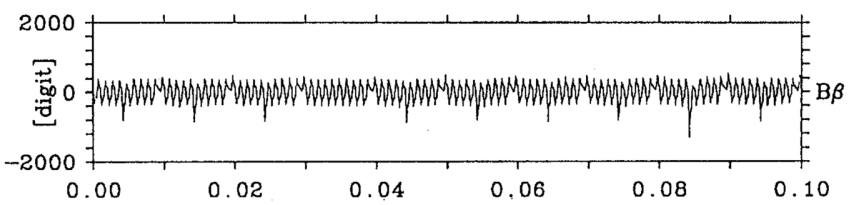

(b)

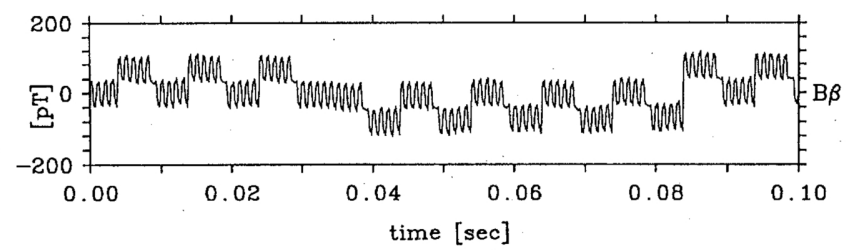

Fig. 14. Example wave forms of the calibration signal when it was imposed on a search coil ( $\beta$ axis) on October 13, 1992. (a) The wave form directly generated from telemetry data of the WFC. This wave form suggests that the original calibration signal was deformed when it passed through the pre-amplifier and the receiver circuit which have non-flat transfer functions (the frequency response of the amplitude and of the phase rotation). (b) The wave form when the transfer functions of the receiver circuit including the search coil and its pre-amplifier were taken into account in the restoration procedure. 
form after the transfer function for the entire circuit (from the pre-amplifier to the WFC receiver) was taken into account in the restoration process. The processed wave form restores the data to the original known input wave form of the calibration signal.

Since the calibration signal is applied to the electric dipole antennas via a small resistance, the potential drop in the plasma medium can be neglected. On the other hand, by applying the calibration signal to the antenna elements via a large resistance, the potential drop in the plasma medium can not be neglected. This is because the antenna sheath impedance can become comparable to the large value of the resistance used. Using this effect, the sheath impedance formed around the electric antenna elements can be estimated from differences in the amplitude and phase for two different values of the resistance. The equivalent circuits shown in Fig. 4 for the electric antenna when it is immersed in a plasma are used to the estimate the antenna sheath impedances $\left(Z_{\mathrm{shw}}\right.$ and $Z_{\mathrm{shp}}$ for the WANT and the PANT, respectively in the figure) and the absolute value of the wave electric field.

\section{Pre-Flight Calibration}

For precise estimations on the absolute values of the electric and magnetic fields, all the gain and phase rotation for every circuit of the receiver system were measured as a function of frequency and input/output amplitude characteristics in the pre-flight calibration test.

Since the PWI has five pre-amplifiers and three types of receiver systems, a large amount of calibration data were obtained and archived for later use in the analysis of the observed data. For calibrating of the SFA and the MCA data, only frequency responses of the gain and the input/output amplitude characteristics are used. However, for the analysis of the WFC data the phase rotation as a function of frequency is very important information in addition to the amplitude frequency response. The majority of the calibration data were obtained by spectrum analyzers and other digital equipment which allowed them to be directly stored into computer files during the ground calibration tests. However, some of the data, by necessity, was made on analog equipment (i.e. photo-camera) and the measured values from these data digitized manually, then stored in computer files.

\subsection{Frequency characteristics of pre-amplifiers}

\subsubsection{For electric field}

As shown in Fig. 4, each dipole antenna (WANT and PANT) is connected to both EFD and PWI pre-amplifiers. These pre-amplifiers are designed to operate independently without disturbing their counterparts.

Examples of the frequency responses of the gain for the WANT and PANT pre-amplifiers used in the PWI (hereafter termed WANT-Pre and PANT-Pre, respectively) are shown in Figs. 15(a) and (b). These figures show the lower limits of the passing frequencies to be $3.6 \mathrm{~Hz}$ and 2.4 $\mathrm{Hz}$ (WANT-Pre and PANT-Pre respectively) as described in Subsections 2.2.1 and 2.2.2. The important difference point between (a) and (b) is the gain in the mid-frequency range of flat response. The PANT-Pre exhibits a $-5.6 \mathrm{~dB}$ gain, while the WANT-Pre provides a $-0.6 \mathrm{~dB}$ gain. This difference is caused by differences in the signal attenuation for the input circuits of the WANT and PANT pre-amplifiers. Here the ratio of the decoupling capacitance to the stray capacitance formed in the input terminal for the two pre-amplifiers is different. Figures 16(a) and (b) show the phase rotations as a function of frequency for both these pre-amplifiers. The phase rotation effect in the high pass filter circuits having cutoff frequencies of 3.6 and $2.4 \mathrm{~Hz}$ is seen in each figure and last up to $500 \mathrm{~Hz}$. In order to precisely estimate the field polarizations from the captured wave forms, these phase rotation effects must always be included. Calibration data for frequencies up to $1 \mathrm{MHz}$ have been acquired for every decade in frequency. 
(a)

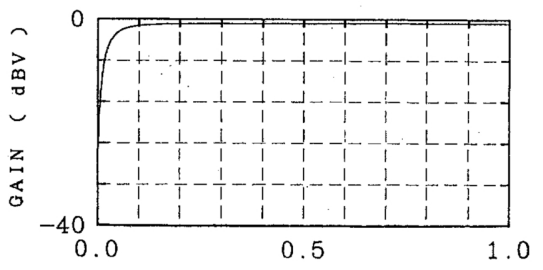

(b)

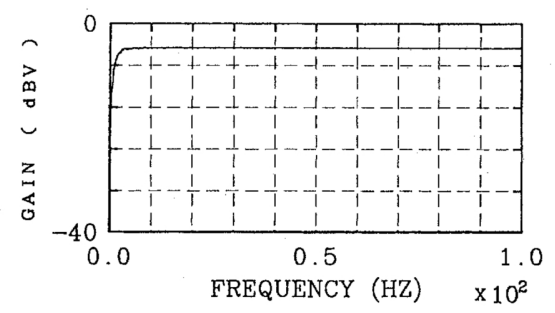

Fig. 15 (a)

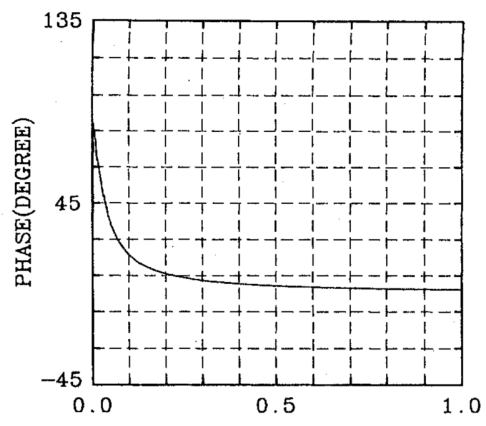

(b)

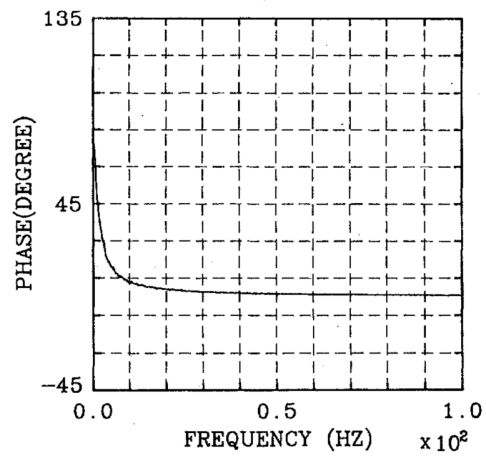

Fig. 16

Fig. 15. An example of the frequency responses of the gain in a very low frequency range of (a) the WANT and (b) the PANT pre-amplifiers. High-pass filtering characteristics determined by a decoupling capacitance and an input resistance connected between the antenna element and the pre-amplifier are found in the frequency range below $10 \mathrm{~Hz}$. Gain values in the flat frequency response regions are $-0.6 \mathrm{~dB}$ for the WANT and -5.6 $\mathrm{dB}$ for the PANT. The difference is caused by the difference on the amplitude attenuation in the input circuits (combination of the decoupling capacitance and a stray capacity formed at the input terminal) for both the pre-amplifiers.

Fig. 16. Examples of phase rotation data as a function of frequency in a very low frequency range in (a) WANT and (b) PANT pre-amplifiers. The steep phase rotations in the lower frequency range are due to the high-pass filtering characteristics as described in Fig. 15. Gradual phase rotations are seen up to $500 \mathrm{~Hz}$, however, no phase rotation is found above the frequency up to $1 \mathrm{MHz}$.

\subsubsection{For magnetic field}

Since the search coil is connected directly to the input terminals of the pre-amplifier as shown in Fig. 6, the instrument frequency characteristics must include the total circuit of both the search coil and the pre-amplifier. The frequency response of the gain and the phase rotations for this circuit were already shown in Figs. 7 (a) and (b), respectively. The characteristics of all search coil and pre-amplifier circuits are essentially the same as those shown in this figure.

\subsection{Frequency characteristics of main amplifiers}

A large amount of calibration data for the main amplifiers was obtained because the electrical characteristics of the receivers are different for different observation modes.

The quantities measured in the calibration effort are summarized as follows:

1. The input/output amplitude characteristics at selected frequencies for all eight SFA receivers (five Bands for the electric field and three Bands for the magnetic field) in both High and Low gain modes. Figure 17 shows an example of the Input/Output amplitude characteristics 


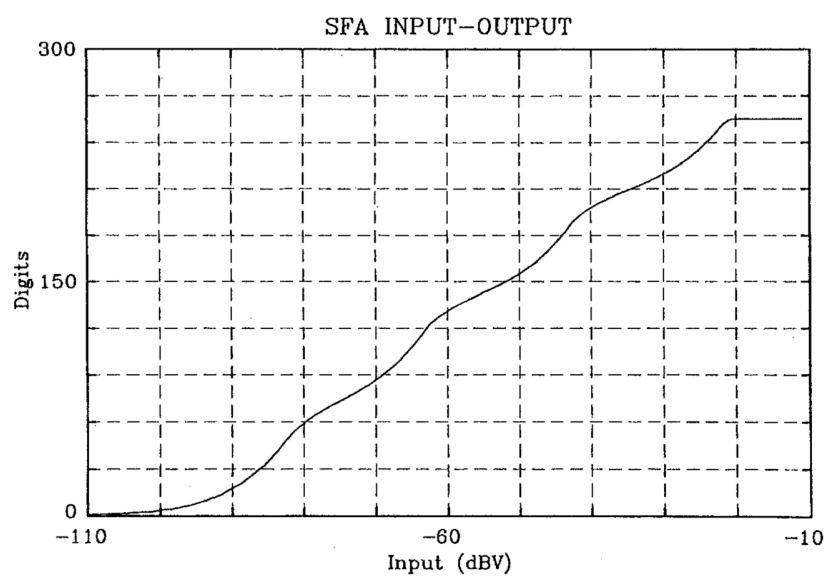

Fig. 17. A representative example of Input/Output amplitude characteristics of an SFA receiver (Band 4 for electric field). In this measurement, the receiver was in the Low gain and the Fixed frequency $(55.67 \mathrm{kHz})$ modes. The characteristic curve shows a dynamic range of $90 \mathrm{~dB}$. Four ripples seen in the characteristic are caused by a cascade connection of four quasi-logarithmic amplifiers.

obtained at a frequency of $55.67 \mathrm{kHz}$ in Band 4 .

2. The frequency responses of the gain in the eight SFA receivers for their High and Low gain modes. Though the example is not shown herein, the frequency responses of the gain are nearly flat over the frequency range in each band as required by the design specification.

3. The input/output amplitude characteristics of the MCA 34 channel analyzer (20 channels for electric and 14 channels for magnetic fields).

4. The WFC calibration data for the two electric and three magnetic field receivers were taken for each of the following operational modes in these calibration measurements both the amplitude and the phase rotations for 48 cases were measured as a function of frequency.

(a) Two telemetry Formats (Format 2 and 3).

(b) Two cutoff frequencies $(10 \mathrm{~Hz}$ and $100 \mathrm{~Hz})$ of the HPF.

(c) Two gains (High and Low).

(d) One Memory mode and two Direct modes (Single and Dual field components).

Figures 18(a) and (b) show the frequency response of the gain and phase rotation for the WFC circuit. An abrupt change of the phase is seen near the lower and upper cut-off frequencies. A calibration table has been constructed from these data. Great care has been taken in its construction since errors due to misreading some of the phase values would certainly cause large errors in the wave polarizations.

\section{Data Processing}

All real time data observed with the GEOTAIL spacecraft are received at the Usuda Deep Space Center (UDSC) and transferred directly to the Sagamihara Space Operation Center (SSOC) at the Institute of Space and Astronautical Science (ISAS) on a real time basis. On the other hand, data retrieved from the data recorder on board the GEOTAIL spacecraft are received and stored onto magnetic tapes at the NASA Deep Space Network (DSN) and sent back to ISAS via the NASA Goddard Space Flight Center (GSFC).

At ISAS, three kinds of data are produced: they are the Quick Look (QL) data, the RAW data and the LEVEL-1 data. The QL data are used for real-time monitoring of the GEOTAIL 
(a)

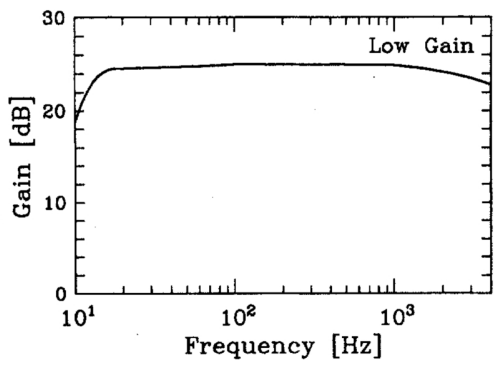

(b)

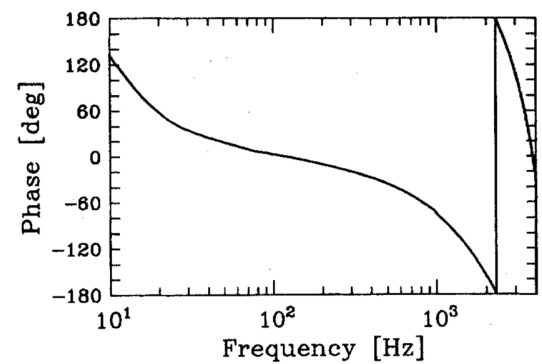

Fig. 18. Frequency responses of (a) gain and of (b) phase rotation for a representative receiver (for $E_{u}$ component) in the WFC. These responses were obtained when the receiver was in Format 2, Low gain, and Memory modes, and the HPF with a lower frequency cutoff of $10 \mathrm{~Hz}$ was selected. Although the gain characteristic is nearly flat between $20 \mathrm{~Hz}$ and $1 \mathrm{kHz}$, the phase exhibits $120^{\circ}$ rotation in the same frequency range, and $520^{\circ}$ rotation between $10 \mathrm{~Hz}$ and $4 \mathrm{kHz}$.

spacecraft during its operations at SSOC. At SSOC, the QL data is produced directly from the telemetry data received, then continuously stored in a file. These data do not include information on the spin phase of the spacecraft and the correct time of the observations. The RAW data are produced just after the end of each period of observation at UDSC and these data are used for a delayed look (thus termed DL). They do not include information on the spin phase and the correct time of the observation either. The LEVEL-1 data are produced from the database named "SIRIUS" almost one week after observation. Since these data include the spin information and the correct observation time, they can be used for scientific analysis. LEVEL-1 data are stored in a data storage system named "FIREX" at ISAS.

All PWI data are transferred from ISAS to the Radio Atmospheric Science Center (RASC) at Kyoto University through the internet network. After calibrating of the wave amplitudes and phases, the data are reduced to bit pattern data which have an X-Window Dump (XWD) format of dynamic frequency spectra ( $f$ - $t$ diagram) for both the electric and magnetic field components. The XWD data and the original data (the LEVEL-1 data) are distributed to the PWI co-investigators (Co-Is) at their request. PWI Co-Is can access these data stored in the magneto optical disks through the internet network.

In the following sections, the data transfer from ISAS to RASC and the data processing at RASC are described.

\subsection{Data transfer from ISAS to RASC}

In the initial operational phase of the mission which extended to five months after the GEOTAIL launch, the QL and the LEVEL-1 data were produced at ISAS, then transferred to RASC through two dedicated telephone lines. Currently the telephone lines have been replaced with an academic TCP/IP network with a usable bit rate of $512 \mathrm{kbps}$. A work-station named "gtlpwi1" is installed at ISAS and acts as a gateway for the transfer of the PWI data. When the LEVEL-1 data are newly generated on the FIREX storage system, the "gtlpwi1" automatically copies them from the FIREX to its hard disk storage. After compressing the LEVEL-1 data, the "gtlpwil" starts the transfer of the data to RASC through the network.

In the near future, an ISDN communication line will be set up between ISAS and RASC as a backup system for the academic TCP/IP network system.

\subsection{Flow chart for PWI data processing}

The LEVEL-1 data received at RASC are first decompressed and then the XWD data are created for displaying $f$ - $t$ diagrams in the $\mathrm{X}$-window format. Dynamic frequency spectra $(f-t$ 
diagram) of the SFA data are then plotted on an X-window terminal. These procedures are conducted automatically at RASC with the aid of a program library described below.

The program library for the PWI data processing has been built through the efforts of individuals at RASC, Kyoto University, Kanazawa University and Toyama Prefectural University. The library consists of three groups of programs named "GTLGET", "GTLX", and "GTLGRPH" (see flow charts shown in Fig. 19). The GTLGET library is a group of programs used to read the data in the three different types of formatted data (QL, RAW and LEVEL-1). The GTLGET can read not only the PWI science data but also the PWI house keeping data, spin information and calibrated values of the flux-gate magnetometer data provided from the MGF. The GTLX library is the second program group and is a graphic package used for plotting the data on X-Window display systems. The third program group is the GTLGRPH library which consists of utility programs used for the design and display of graphic data on a CRT display. After creating the XWD data for the SFA and the MCA, we store the LEVEL-1 data onto $8 \mathrm{~mm}$ tapes for later analysis and also for archival purposes.

While these library programs are written in the $\mathrm{C}$ programming language, they can be called from FORTRAN programs which allows access to scientists who may not be familiar with the C language.

\subsection{Display formats of $f$-t diagram}

The displayed image patterns in the XWD format are stored on magneto-optical disk. The standardized displaying format can be utilized for the QL data and for the calibrated data made from the LEVEL-1 data. Image formats with 8 bit pseudo colored planes were also developed for the XWD data file. As a diagnostic, furthermore, the electron cyclotron frequency calculated from the DC magnetic field intensity (supplied by the MGF team) is plotted together with the PWI data in the $f$ - $t$ diagrams. The cyclotron frequency provides an important physical reference used in identification of the spacecraft location.

A standard time-span for a set of $f$ - $t$ diagrams consisting of SFA electric and magnetic field data is $100 \mathrm{~min}$. The number of picture cells (PIXELs) in the X-window format is 990 dots (in

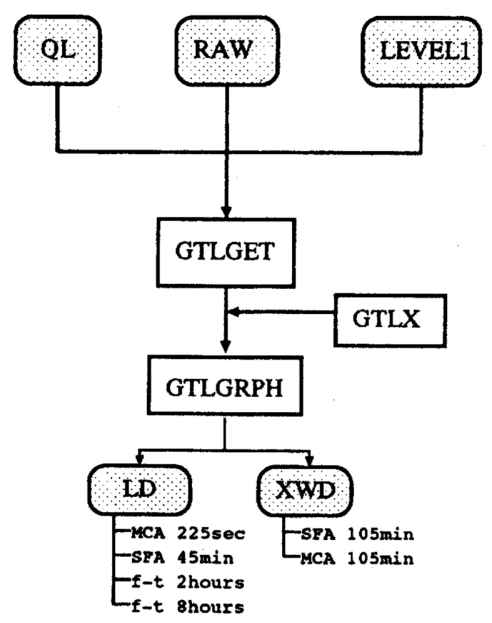

Fig. 19. Flow chart for the PWI data processing. Three kinds of data, Quick Look (QL), RAW, and LEVEL-1, are provided from ISAS. They are read out by a program library of GTLGET, then they are transformed to graphic data by the GTLGRPH library, and XWD data are finally created for the display of $f$ - $t$ diagrams in the X-Window format. Laser Disk (LD) is used for a quick review of the $f$ - $t$ diagram. 
the horizontal direction) $\times 680$ dots (in the vertical direction). This means that in the vertical direction, one picture cell displays the average value of four frequency components, whereas in the horizontal direction, one picture cell consists of a $8.8 \mathrm{sec}$ observation interval. As for the MCA data, the similar set of $f$ - $t$ diagrams is $45 \mathrm{~min}$ in duration even though the sampling interval of the MCA data is $0.25 \mathrm{sec}$. Thus 6 or 7 XWD data files are generated for the SFA along with 15 files for the MCA for the data from about 10 hours of observation. For the DSN data from 24 hours of observation, about 16 SFA XWD data files and $32 \mathrm{MCA}$ files will be generated from this data.

At present, a set of survey $f$ - $t$ diagrams corresponding to 12 hours of observation are also generated in order to monitor macroscopic wave behaviors and provide an overview of the data. These 12-hour $f$ - $t$ diagrams are constructed mainly from the SFA data, but the three lowest frequency channels $(5.6 \mathrm{~Hz}, 10 \mathrm{~Hz}$ and $17.8 \mathrm{~Hz})$ of the MCA data are also included below the lowest frequency $(24 \mathrm{~Hz})$ SFA data in order to supplement the low frequency resolution.

\subsection{Laser Disk system}

A Laser Disk (LD) is used for a quick review of the $f$ - $t$ diagrams (45 minutes plot for SFA and $225 \mathrm{msec}$ plot for MCA) from both the electric and magnetic fields. The XWD data are converted to a video signal having the NTSC format and stored on the laser disk which can archive 110,000 video frames. Although the quality of the video pictures on a TV screen is not good when compared with the XWD pictures on the computer terminal, the laser disk system allows us to store a large amount of graphical data on a media which provides parallel access to arbitrary picture frames quickly.

\subsection{Coordinate systems}

The orbit and the attitude of the GEOTAIL spacecraft are defined in either the Geocentric Solar Ecliptic (GSE) or the Geocentric Solar Magnetic (GSM) coordinate systems. Wave characteristics such as wavenormal direction, polarization direction and Poynting vector must also be determined in these systems. Wave electric and magnetic field vectors are transformed from spacecraft coordinates into GSE and GMS using the appropriate transformation matrices. Additionally, the five wave forms of the electric and magnetic field components which are simultaneously observed by the WFC also need to be transformed into their equivalent values in the GSE or the GSM coordinate systems.

Since the GEOTAIL spacecraft is rotating clockwise around the $Z$ axis $\left(Z_{\mathrm{gtl}}\right)$ in the spacecraft coordinate system (see Fig. 2) with a spin period of almost $3 \mathrm{sec}$, the instantaneous values of the field components detected by the sensors must be converted to their value in the GSE or GSM coordinate systems in accordance with the attitude of the spinning spacecraft. Under current procedure the static magnetic field vector data are released from MGF group. In their conversion, the GEOTAIL spacecraft spin axis $\left(Z_{\mathrm{gtl}}\right)$ is considered to be parallel to the $Z_{\mathrm{GSE}}$ in the GSE coordinate axis even though the spin axis is tilted toward the sun by angle of about $2.7^{\circ}$. The $Z_{\mathrm{gtl}}$ axis is tilted sunward so that shading of the WANT and PANT antenna elements by the spacecraft body is minimized in order to mitigate imbalanced electric potentials between the two elements making up dipole antenna. Such imbalanced electric potentials could arise as shading of an antenna element modifies the photo-electron emissions from its surface from that of its counterpart thereby imbalancing the dipole.

The coordinate transformation matrix for the electric field is given by

$$
\left(\begin{array}{c}
E_{X \mathrm{GSE}} \\
E_{Y \mathrm{GSE}} \\
E_{Z \mathrm{GSE}}
\end{array}\right)=\left(\begin{array}{ccc}
\cos \theta_{\mathrm{E}} & -\sin \theta_{\mathrm{E}} & 0 \\
\sin \theta_{\mathrm{E}} & \cos \theta_{\mathrm{E}} & 0 \\
0 & 0 & 1
\end{array}\right)\left(\begin{array}{c}
E_{U} \\
E_{V} \\
E_{Z}
\end{array}\right)
$$


where

$$
\theta_{\mathrm{E}}=\theta_{0}+\phi_{X-U}+V_{\text {spin }} \times \delta t \times n .
$$

The quantity $\theta_{0}$ is the spin phase angle of the $X_{\text {gtl }}$ axis measured from the $X_{\mathrm{GSE}}$ axis at an arbitrary time $t_{0}$. This spin phase angle is provided in each frame of the LEVEL-1 data. The quantity $\phi_{X-U}\left(=-15^{\circ}\right)$ is the phase angle of the $U_{\text {gtl }}$ axis measured from the $X_{\text {gtl }}$ axis and $V_{\text {spin }}$ is the angular frequency (degree/sec) of the spin rotation. Here, $\delta t$ is the data sampling interval and $n$ represents the successive data number counted from the time $t_{0}$.

For the magnetic field, we use a similar transformation matrix of the form

$$
\left(\begin{array}{c}
B_{X \mathrm{GSE}} \\
B_{Y \mathrm{GSE}} \\
B_{Z \mathrm{GSE}}
\end{array}\right)=\left(\begin{array}{ccc}
\cos \theta_{\mathrm{B}} & -\sin \theta_{\mathrm{B}} & 0 \\
\sin \theta_{\mathrm{B}} & \cos \theta_{\mathrm{B}} & 0 \\
0 & 0 & 1
\end{array}\right)\left(\begin{array}{c}
B_{\alpha} \\
B_{\beta} \\
B_{\gamma}
\end{array}\right)
$$

where

$$
\theta_{\mathrm{B}}=\theta_{0}+\phi_{X-\alpha}+V_{\text {spin }} \times \delta t \times n .
$$

The quantity $\phi_{X-\alpha}\left(=-45^{\circ}\right)$ indicates the phase angle of the $\alpha$ direction measured from the $X_{\text {gtl }}$ axis in the spacecraft coordinate system.

\section{Initial Results}

The PWI began making observations after the deployment of the electric antennas on August 27, 1992 and the magnetic sensors on September 16, 1992. In this section, we present some of the initial results of the PWI observations.

\subsection{An effect of photo-electron emissions}

Ringing noise is present in the electric field data obtained by the WFC instrument as shown in Fig. 20. The period of the ringing noise coincides with the half spin period of the spacecraft and the sense of potential change in the ringing wave forms reverses for the successive ringing every half spin period. The difference in the occurrence phase of this ringing between the WANT and PANT data is $90^{\circ}$. In efforts to understand this effect, the shaded area on the antenna element has been examined in relation to the spin phase angle of the GEOTAIL spacecraft. However, no

\section{GEOTAIL PWI WFC}

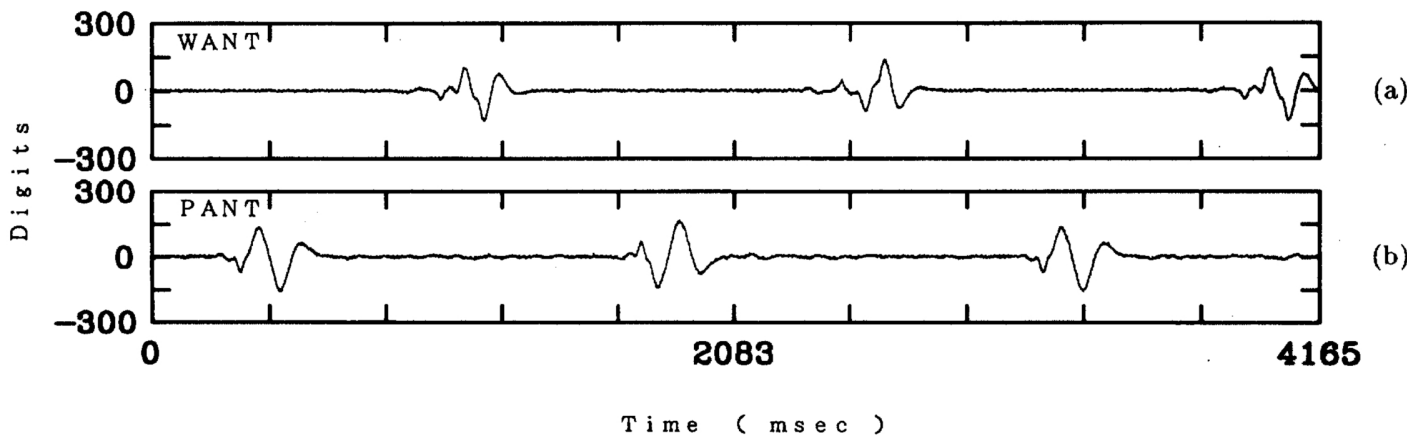

Fig. 20. Wave forms of ringing noise detected by (a) WANT and (b) PANT. The ringing noise is observed every half spin period in each dipole antenna. The difference in the occurrence phases of the spiky noise between the WANT and PANT data is $90^{\circ}$. In each ringing wave form, the sense of electrical potential change is opposite to that in the successive the ringing noise occurring in every half spin period. 
correlation has been found between the time rate of change of the estimated shaded area on an antenna element and the observed ringing wave forms. These wave forms are thought to be the result of ringing when a discontinuous or abrupt change in potential is applied to an electric circuit having a time derivative characteristic. Thus the reversal of the sense of the ringing in successive forms suggests that the sense of the discontinuous or abrupt change in potential is also reversed every half spin period. Such a change in potential is thought to be generated in the following manner. In general, the potential of an element for a dipole antenna is increased by photoelectron emission when solar UV irradiates the surface of the element. The EFD group (Tsuduki et al., 1993) has demonstrated observationally the sinusoidal potential variation ( $1 / 2$ period) for one element when the element rotates from the sunward to the anti-sunward direction as shown schematically in Fig. 21(a). The evident potential variation can be explained by considering the variation in solar UV power density for the sunlit area on the surface of the element wire in relation with the spacecraft rotation. However, we have to pay particular attention to values in phase angle around $0^{\circ}\left(360^{\circ}\right)$ and $180^{\circ}$, where $0^{\circ}$ means the sunward direction. Since the output signal for a dipole antenna is obtained as the differential potential between two elements directed opposite to one another, the output voltage of a differential amplifier can be obtained by considering the difference between Element-A and Element-B in Fig. 21(a). If the characteristic potential variation on the elements are purely sinusoidal (solid lines in Fig. 21(a)), there emerges no potential difference from the differential amplifier. However, if the potential is different (e.g. higher potential drawn by broken lines in (a)) near $0^{\circ}$ than for position near $180^{\circ}$, there emerges potential difference as shown in Fig. 21(b). Even if this potential difference is extremely small for EFD measurement, WFC can detect the potential difference by observing ringing wave forms as a result of the potential variating signal passing through the electric circuit having the time derivative characteristic. A strong candidate for the generation of the differences in antenna potential near phase angles of $0^{\circ}$ and $180^{\circ}$ comes from imbalanced photo-electron emissions in these two directions of the element. An imbalance in the photo-electron emission for the antenna element can occur in relation to phase angle due to shading from the spacecraft body on the antisunward side. Further, when the antenna element nears phase angles around $180^{\circ}$, about one third of the total length of the element wire is shaded by the high gain telemetry antenna. This result is interesting in that the shading effect occurs even for that part of antenna which is coated

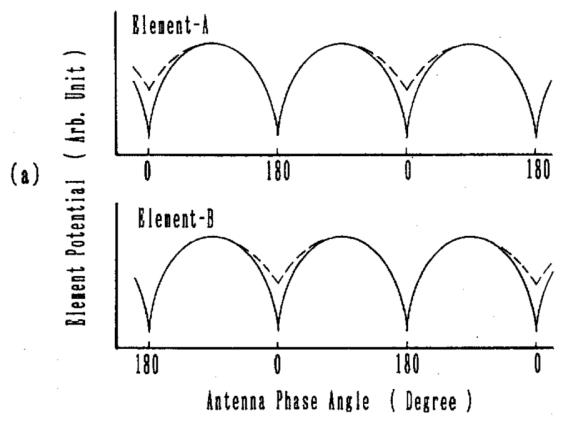

(b)

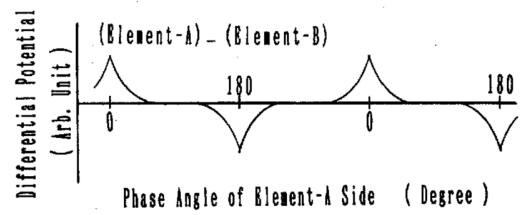

Fig. 21. (a) Schematic showing of the potential variations for Element-A and Element-B measured against the spacecraft body as a function of antenna phase angle, where $0^{\circ}$ and $180^{\circ}$ indicate sunward and anti-sunward directions, respectively, presented by the EFD group (Tsuruda et al., 1994, private communication). The phase angles for the individual elements are $180^{\circ}$ out of phase since they represent of a dipole antenna and are situated on opposite sides of the spacecraft. Observational results show a higher potential as shown by broken lines at $0^{\circ}$ phase angels as compared with that at $180^{\circ}$ (Tsuduki et al., 1993). (b) The output voltage from the dipole antenna, which represents the differential potential between the two elements depicted in (a) having used potential variation illustrated by the broken lines. 
by insulative material. The present result suggests that even insulatively coated antennas might be affected by photo-electron emission processes. A laboratory simulation is being attempted in which representative insulative coated antenna elements are used in an effort to ascertain the photo-electron emission effects from the insulator.

\subsection{Plasma wave observations}

Two examples of plasma wave observations have been selected for presentation herein. First we will examine the waves observed in conjunction with plasmasheet crossings in the tail region, then those observed during a skimming pass of the dayside magnetopause. Though we need additional time for performing a detailed analysis such as comparison with previous wave observations by IMP-6, IMP-8 and ISEE-3, our intent the initial results to demonstrate the capabilities of the PWI instrument.

(1) Wave spectra for a plasmasheet crossing in the magnetotail on September 18, 1992

The GEOTAIL experienced its first crossing of the plasmasheet in the distant magnetotail around $X=-120$ Re on September 18, 1992 after the PWI observations had commenced on September 8, 1992. The orbit of the GEOTAIL spacecraft in the GSM coordinates from September 8 to October 10, 1992 is depicted in Fig. 22. During this period, the GEOTAIL traversed the distance from $X=-40$ to $-135 \mathrm{Re}, Y=40$ to $-30 \mathrm{Re}, Z=-20$ to 20 Re. In GSM coordinates, the GEOTAIL varied its location on September 18, 1992 through the range from $X=-120$ to $-123 \operatorname{Re}, Y=25$ to $22 \operatorname{Re}, Z=10$ to $1 \mathrm{Re}$. Judging from these variations in location only, the GEOTAIL seems to have traversed the magnetotail region passing through the plasmasheet, the lobe and possibly the magnetosheath or the low latitude boundary layer in the magnetosheath although the spatial region the spacecraft has covered should be confirmed by detailed analysis of magnetic data (all plasma instruments were off on this day).

Figure 23 shows the dynamic frequency spectra measured by the SFA for 2 hours out of the total 7.5 hours of observations made on September 18, 1992. The upper and lower panels are the spectra for the electric $E_{U}$ component and for the magnetic $B_{\gamma}$ component corresponding to plasma waves observed from 1150 UT to 1350 UT. The wave intensity is plotted on a logarithmic scale as indicated by the color bar. Large tick marks in the frequency axis show boundaries in each SFA band. We note that SFA data are plotted in the linear scale in each band. The
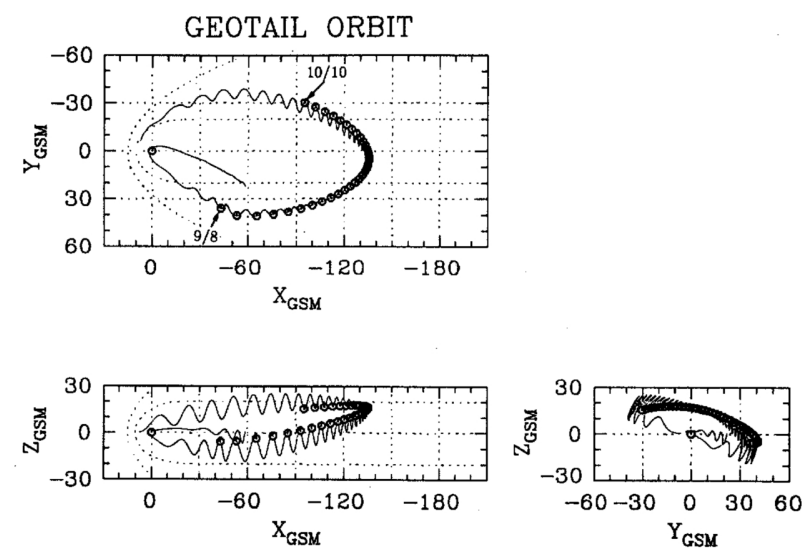

Fig. 22. Orbit trajectory in GSE and GSM coordinates for GEOTAIL spacecraft from September 8 to October 10, 1992. GEOTAIL on September 18 varied its location over the range of $X=-120 \sim-123 \operatorname{Re}, Y=25 \sim 22$ Re, and $Z=10 \sim 1$ Re. 


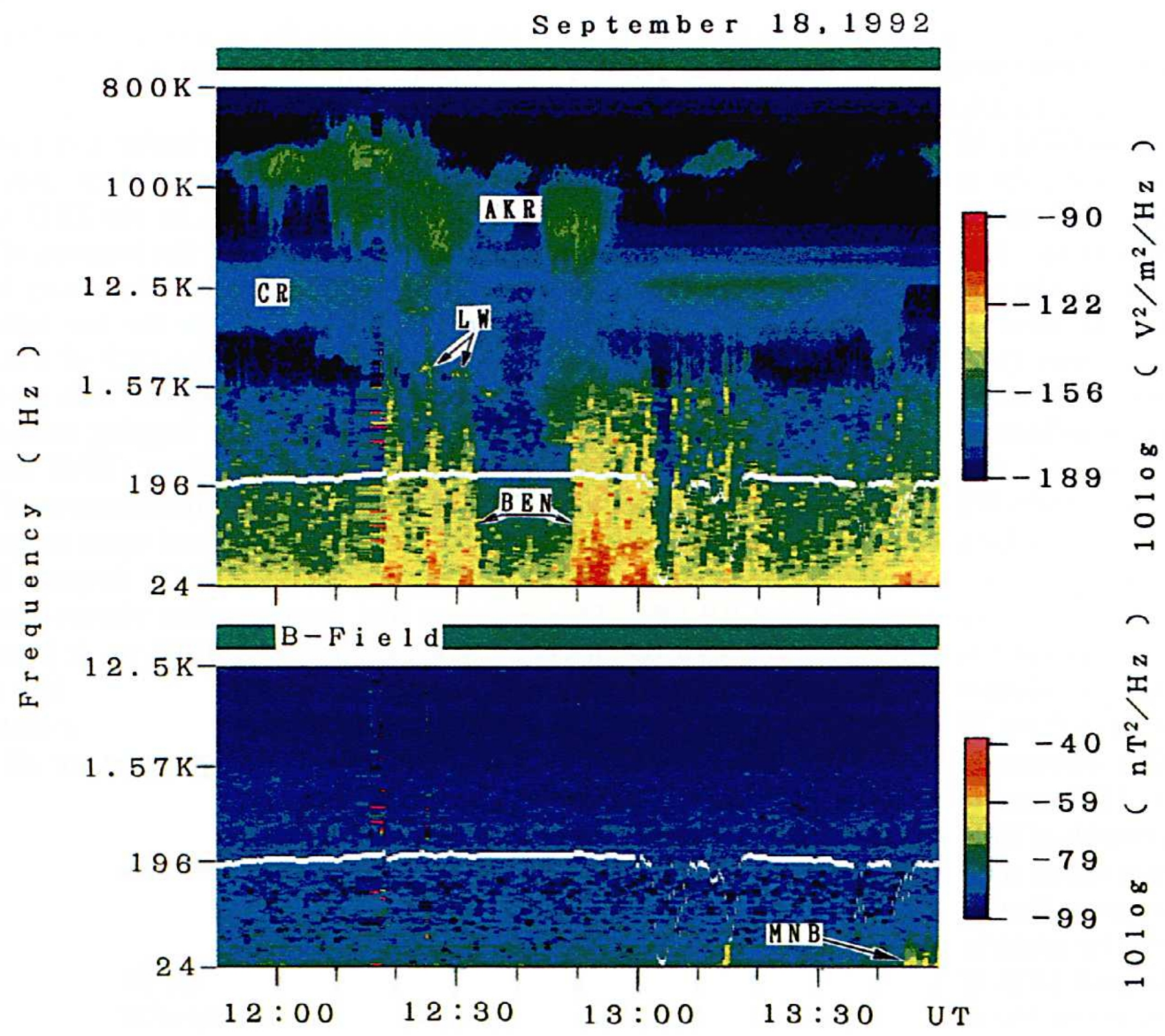

T i me

Fig. 23. Dynamic frequency spectra observed by the SFA for 2 hours out of a total of 7.5 hours of observation on September 18, 1992. The upper and lower panels are the spectra for the electric $E_{U}$ component and for the magnetic $B_{\alpha}$ component of the observed plasma waves from 1150 UT to 1350 UT. The wave intensities are indicated by each color code with a logarithmic scale as shown by the color bars. The white line superimposed in each dynamic spectrum indicates the electron cyclotron frequency calculated from magnetic field intensity provided by the MGF team. The spectra are characterized by rich wave activities such as Auroral Kilometric Radiation (AKR), continuum radiation (CR), Langmuir waves (LW), Broadband Electrostatic Noise (BEN) and Magnetic Noise Burst (MNB).

electron cyclotron frequency calculated from the magnetic field data (provided by the MGF team (Kokubun et al., 1994)) is superimposed on the figure as a white line (the line is actually composed of individual dots of data). The spectra are characterized by enhanced wave activities such as Auroral Kilometric Radiation (AKR), Continuum Radiation (CR), Langmuir Waves (LW), Broadband Electrostatic Noise (BEN) and Magnetic Noise Bursts (MNB). As the SFA has a very fine frequency resolution, we can determine the local electron density with high accuracy by determining the lower cutoff frequency of the continuum radiation or intensified Langmuir waves seen at the lower edge of the continuum radiation. Hereafter we term the lower cutoff frequency 
of the continuum radiation as the "LCF of CR". The electron density determined in this manner is shown in the third panel from the top in Fig. 24. This figure shows the same wave spectra but for a longer time period from 0900 UT to $1630 \mathrm{UT}$ on the same day. The electron density varied from $0.008 / \mathrm{cc}$ to $3.5 / \mathrm{cc}$ (see the third panel in Fig. 24).

As the GEOTAIL particle measurements had not been ready during this initial operation of the spacecraft, the only available measurements, in addition to the plasma wave data, were the magnetic field measured by the MGF team and the electric field measured, by the EFD team (Tsuruda et al., 1994). Without the particle data, it is difficult to establish the location of the spacecraft in the magnetotail such as in the plasmasheet (PS), the plasma sheet boundary layer (PSBL), the lobe (L), the plasma mantle (PM), the magnetosheath (MS) or the low latitude boundary layer (LLBL). However, combining the magnetic field data and the LCF of CR, we have been able to infer the location of the GEOTAIL spacecraft in the tail region. This inferred location is indicated in the lower panel in Fig. 24. Due to the windsock or flapping motion of the magnetotail, the GEOTAIL spacecraft passed through the north plasmasheet (NPS) during 0900 UT to 0950 UT crossing into the North Lobe (NL) at 0950 UT. The identification of the northern or southern with respect to the neutralsheet (NS) was decided based upon magnetic field data (Kokubun, private communication, 1992). At this time the LCF of CR dropped from $8 \mathrm{kHz}$ down to a minimum of about $0.9 \mathrm{kHz}$. This indicates that corresponding plasma density in the plasmasheet was about $0.8 / \mathrm{cc}$ with the density in the lobe at about $0.008 / \mathrm{cc}$. It is noted that though enhanced AKR activity is observed from the beginning of observations on this day, it intensifies from $1000 \mathrm{UT}$ to $1029 \mathrm{UT}$. Along with this intensification the frequency band of the AKR increased especially to lower frequency. This same behavior is observed for all the intense AKR measured on this day. Such broadband spread in frequency seems to be a universal characteristic of the GEOTAIL PWI data have seen so far. It is also interesting that the continuum radiation shows a higher intensity from 1005 UT to 1020 UT when the AKR is most intensified. This suggests that the sources for these two different wave emissions may be somehow interrelated (possibly by particle injection).

Around 1030 UT, the GEOTAIL crossed into the (north) Low Latitude Magnetosheath (LLMS) where the LCF of CR is $15.5 \mathrm{kHz}$ and corresponds to a plasma density of $3 / \mathrm{cc}$. The LCF of CR from 1030 UT to 1140 UT was constant suggesting a fairly stable plasma density of $3 /$ cc. During this time, both electric and magnetic noise bursts are seen ranging from $24 \mathrm{~Hz}$ (which is the minimum frequency detectable by PWI-SFA) to about $8 \mathrm{kHz}$ for the electric measurements and from $24 \mathrm{~Hz}$ to about $80 \mathrm{~Hz}$ for the magnetic measurements. The electric measurements can be referred to as broadband electrostatic noise (BEN) and the magnetic components are termed Magnetic Noise Bursts (MNB). These waves have been measured previously and are characteristic of typical wave emission found in the magnetosheath. The BEN and MNB are quiet for a short time during the period from 1105 UT to $1115 \mathrm{UT}$.

Around 1140 UT, the GEOTAIL entered back into the north plasmasheet (NPS). It appears that the GEOTAIL may have passed through a wavy structure on the dusk side of the magnetotail between the LLMS and the PS since we see three brief dips in the plasma density just before the GEOTAIL entered into the plasmasheet at 1140 UT. Such wavy structure could be caused by a Kelvin-Helmholtz instability at the magnetopause boundary. If this observation is $\mathrm{K}-\mathrm{H}$ instability then it is interesting to observe the K-H instability so far down tail (around $120 \mathrm{Re}$ ). An alternative view is that the spacecraft measured a simple flapping motion of the tail with a time period of about $10 \mathrm{~min}$. Beyond this time the GEOTAIL stayed in the north plasmasheet from $1145 \mathrm{UT}$ to $1207 \mathrm{UT}$. The LCF of CR during this period is fairly constant around $8 \mathrm{kHz}$ indicating the plasma density in the plasmasheet to be about $0.8 / \mathrm{cc}$. At $1207 \mathrm{UT}$, the LCF of $\mathrm{CR}$ abruptly dropped from $8 \mathrm{kHz}$ to $5 \mathrm{kHz}$, indicating an abrupt drop in the plasma density from $0.8 / \mathrm{cc}$ to $0.3 / \mathrm{cc}$. This presumably indicates that the GEOTAIL entered the north lobe (NL) and remained there until $1253 \mathrm{UT}$ when it reentered the plasmasheet again at $1253 \mathrm{UT}$. In 
the lobe, the plasma density dropped gradually from $0.3 / \mathrm{cc}$ to the minimum density of $0.008 / \mathrm{cc}$ at $1249 \mathrm{UT}$ (LCF of CR was about $900 \mathrm{~Hz}$ ). Additionally, the GEOTAIL may have passed through the PSBL from 1207 UT to 1238 UT. The plasma density in the PSBL decreased from $0.3 /$ cc to $0.05 /$ cc. During the passage of the PSBL, three bursts of strong BEN were observed at $1215 \mathrm{UT}, 1227 \mathrm{UT}$ and $1231 \mathrm{UT}$, respectively. No magnetic components were associated with the BEN bursts. It is interesting to note the AKR activity at higher frequencies from $200 \mathrm{kHz}$ to $700 \mathrm{kHz}$ which is enhanced for about ten minutes around $1200 \mathrm{UT}$ and lasts until $1255 \mathrm{UT}$ prior to the onset of the BEN. In association with the BEN emissions, strong Langmuir waves at the plasma frequency are also excited. The observation of the Langmuir waves suggests the presence of an electron beam to provide free energy for generating these waves in the PSBL. It is possible that the GEOTAIL has passed through the northern edge of a plasmoid which consists of multiple magnetic islands. The magnetic field measurements at this time showed (Kokubun and Yamamoto, private communication, 1992) that the direction of the magnetic field tilted by approximately 20 degrees northward at the time of each BEN burst and 20 degrees southward at the rear end of each burst. Such correlated behavior is observed in succession three times. Similar events were also seen during the time period from 1250 UT to 1301 UT. Two bursts of BEN, not well separated, are seen in the electric field spectra. It is not clear whether or not the spacecraft was in the PSBL during this period of observation because of the difficulty in reading the LCF of $\mathrm{CR}$. However, judging from the fact that the LCF of CR jumped up to $8 \mathrm{kHz}$ (corresponding to $0.8 / \mathrm{cc}$, the same density level in the plasmasheet) just at the termination of the bursts at 1301 UT and from the change in the potential of a single probe measured by the EFD team (Tsuruda, private communication, 1992), we can infer that the spacecraft traversed the PSBL at this time. The magnetic field variation, however, did not show a clear signature of a plasmoid passage. The magnitude of the magnetic field showed a slight decrease in total intensity with a single short pulse increase in its inclination (Kokubun and Yamamoto, private communication, 1992). Right after the return to the PS, from 1303 UT to 1306 UT, the GEOTAIL passed through the neutral sheet (NS) where the total magnetic field fell almost to zero. There was a complete lack of wave emissions extending from $50 \mathrm{~Hz}$ to $8 \mathrm{kHz}$ during this period. This quiet feature of the NS has been reported by previous spacecraft observation (Anderson, 1984). After passing through the neutral sheet, the GEOTAIL entered the south plasmasheet (SPS) until 1325 UT. For about 6 minutes from $1325 \mathrm{UT}$, the plasma density dropped down to $4 \mathrm{kHz}$ (corresponding plasma density of $0.2 / \mathrm{cc}$ ). Again we see the intensified Langmuir waves at the edge of the LCF of CR. Evidently the spacecraft was in the edge of the lobe or in the PSBL during this period. Surprisingly, we do not see any BEN, but intense Langmuir waves at the local electron plasma frequency. If the intense Langmuir waves are an indication of the existence of a local electron beam, this may indicate that BEN does not necessarily need an electron stream. Of additional importance is the fact that AKR activity at and prior to this time was non-existent which differs from the previous example of strong BEN.

It seems that the GEOTAIL reentered the northern magnetosheath passing through the magnetopause from 1343 UT to 1353 UT. The magnetic components of the waves below $50 \mathrm{~Hz}$ were enhanced during this time period.

(2) Wave spectra from dayside magnetopause skimming on October 17-18, 1992

Before transferring to a distant tail orbit by means of a double lunar swing-by, the GEOTAIL spacecraft experienced its first dayside magnetopause skimming on October 17 to 18, 1992. Figure 25 shows the GEOTAIL orbit on these days in GSM coordinates. Since the semi-circular orbit ( $\sim 10 \mathrm{Re})$ in the dayside magnetopause skimming was close to a geo-synchronous one, the UDSC was able to accomplish receipt of the telemetry data from GEOTAIL for about 24 hours. The outer and inner dotted lines indicate the expected boundaries of the bow shock and the magnetopause, respectively. The orbital trajectory suggests that the GEOTAIL spacecraft moved from the dawn side of the northern hemisphere to the dusk side of the southern hemisphere while 


\section{GEOTAIL PWI}

'9209.18

\section{SFA}
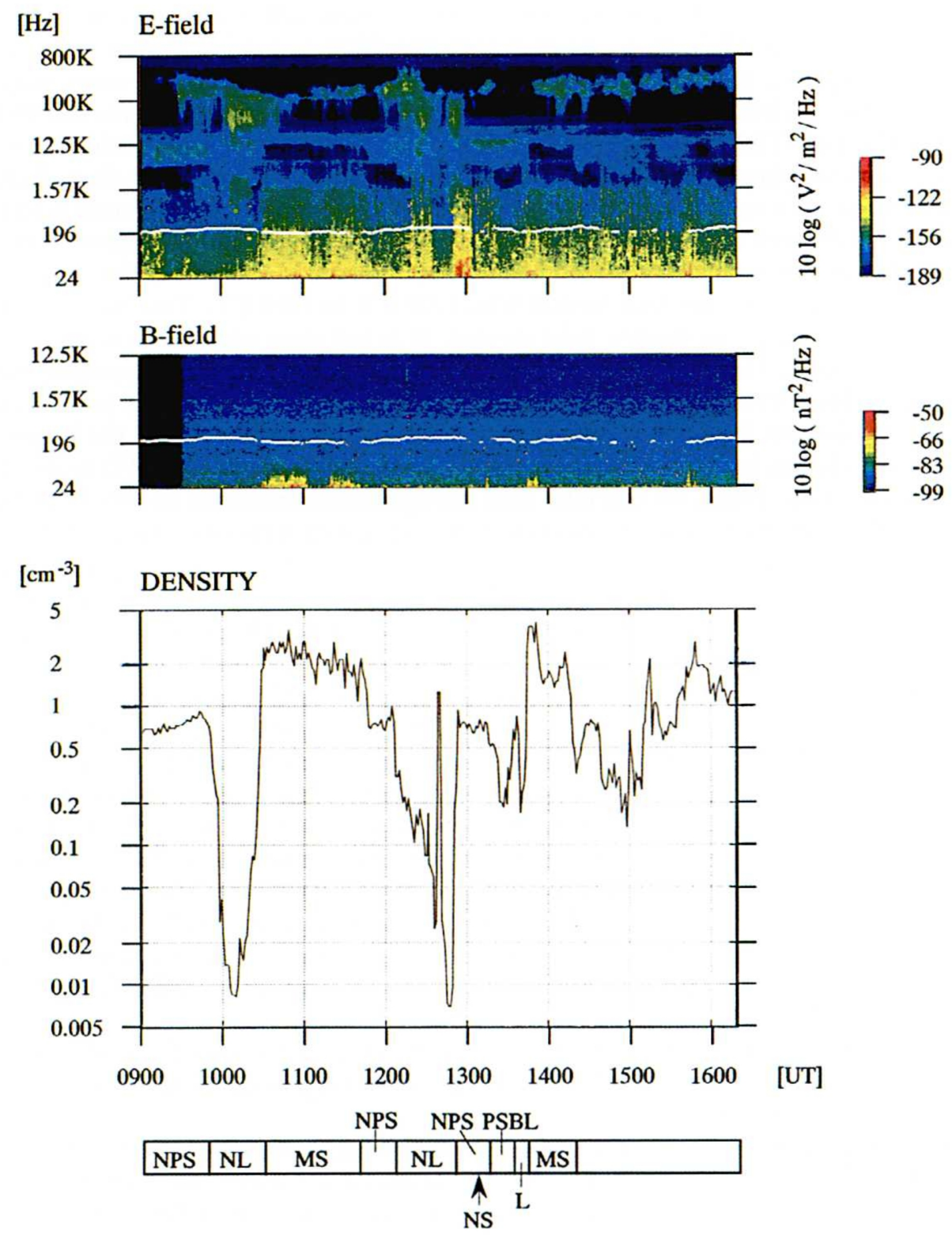

Fig. 24. Dynamic frequency spectra for electric and magnetic fields observed by SFA for 7.5 hours on September 18,1992 (top two panels), electron density profile obtained from the LCF of CR (the third panel), and location of GEOTAIL (the bottom frame) inferred from the spectral features and aided by the magnetic field data. 

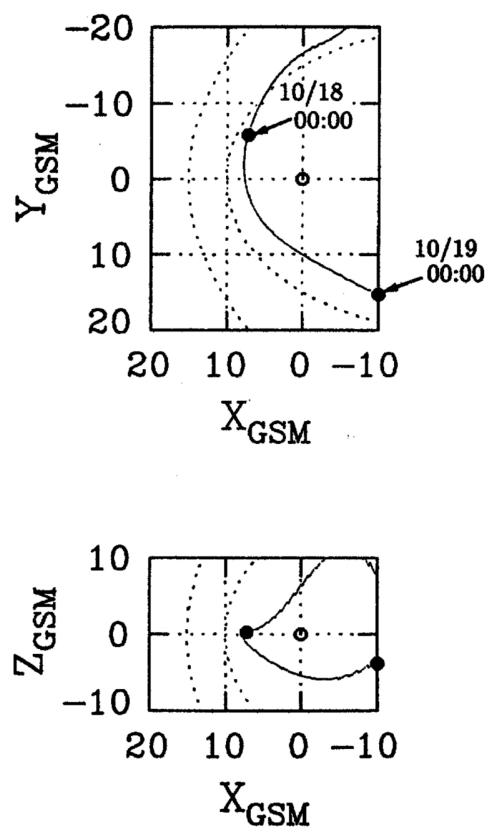

Fig. 25. GEOTAIL orbit in GSM coordinates for October 17 18, 1992. The outer and inner dotted lines indicate the expected boundary of the bow shock and the magnetopause, respectively. GEOTAIL moved from the dawn side of the northern hemisphere to the dusk side of the southern hemisphere while skimming the dayside magnetopause.

skimming portions of the dayside magnetopause.

Figure 26 shows an $f$ - $t$ diagram of the SFA data for about 24 hours during this period. The upper panel shows the electric $E_{V}$ component and the lower one shows the magnetic $B_{\gamma}$ component. The white dotted line indicates the electron cyclotron frequency calculated from the DC magnetic field intensity provided by the MGF team. The spectral structure found in these figures can be divided into three different periods: The first period is from 1510 UT to $2100 \mathrm{UT}$ on October 17, the second one extends from 2100 UT on October 17 to 0800 UT on October 18, and the third one is from 0800 UT to 1415 UT on October 18.

During the first period, GEOTAIL traversed the inner magnetosheath region. The background electron density calculated from the LCF of CR is $20 / \mathrm{cc}$, and the magnetic field appears very turbulent. Intense electromagnetic waves are also observed extending in frequency up to $200 \mathrm{~Hz}$ for both the electric and magnetic field components. These are observations exhibit the characteristic features of wave emissions in the magnetosheath. From 1800 UT, the PWI observed repetitions of a sudden decrease then increase in the LCF of CR. This suggests that the GEOTAIL was repeatedly crossing the magnetopause and moving in and out of the magnetosphere. This would suggest that the magnetopause has a wavy structure which may be caused by a Kelvin-Helmholtz instability. Further, such wavy structure might connect to the tail region as described in the previous example observed on September 8.

At the beginning of the second period mentioned above, GEOTAIL entered into the magnetosphere. As time goes on for this period, the background electron density gradually increases from $0.3 / \mathrm{cc}$ to $1.5 / \mathrm{cc}$. From the spectral features of the wave activity, this period can be further divided into three intervals, 2100 UT to $2300 \mathrm{UT}, 2300 \mathrm{UT}$ to $0500 \mathrm{UT}$ of the next day, and $0500 \mathrm{UT}$ to $0800 \mathrm{UT}$. Judging from a little fluctuation in the ambient magnetic field during the 


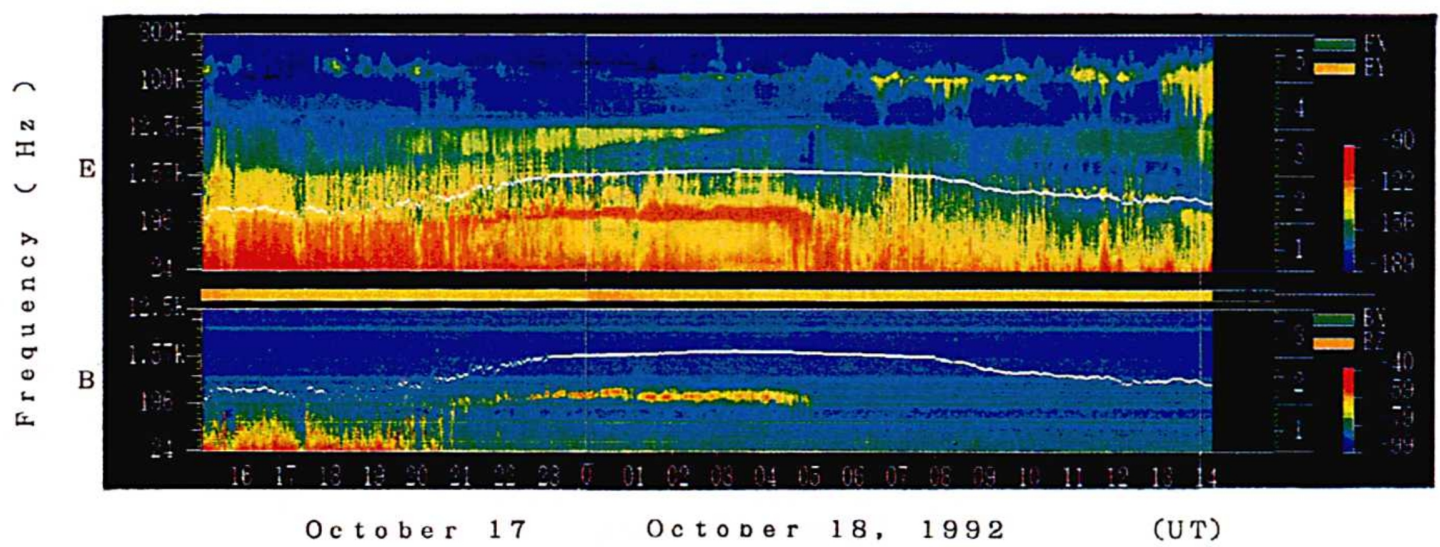

Fig. 26. Dynamic frequency spectra measured by the SFA for about 24 hours on October 17 to 18,1992 when the GEOTAIL was skimming the dayside magnetopause. The upper and lower panels are spectra for the electric $E_{V}$ component and for the magnetic $B_{\gamma}$ component from the observed plasma waves. The white dotted line indicates the electron cyclotron frequency. The spectral features suggest that the GEOTAIL spacecraft has traversed three different regions during the time periods of 1510 UT to 2100 UT on October 17, 2100 UT on October 17 to 0800 UT on October 18, and 0800 UT to 1415 UT on October 18.

\section{$\mathrm{ECH}$ (Electron Cyclotron Harmonics)}

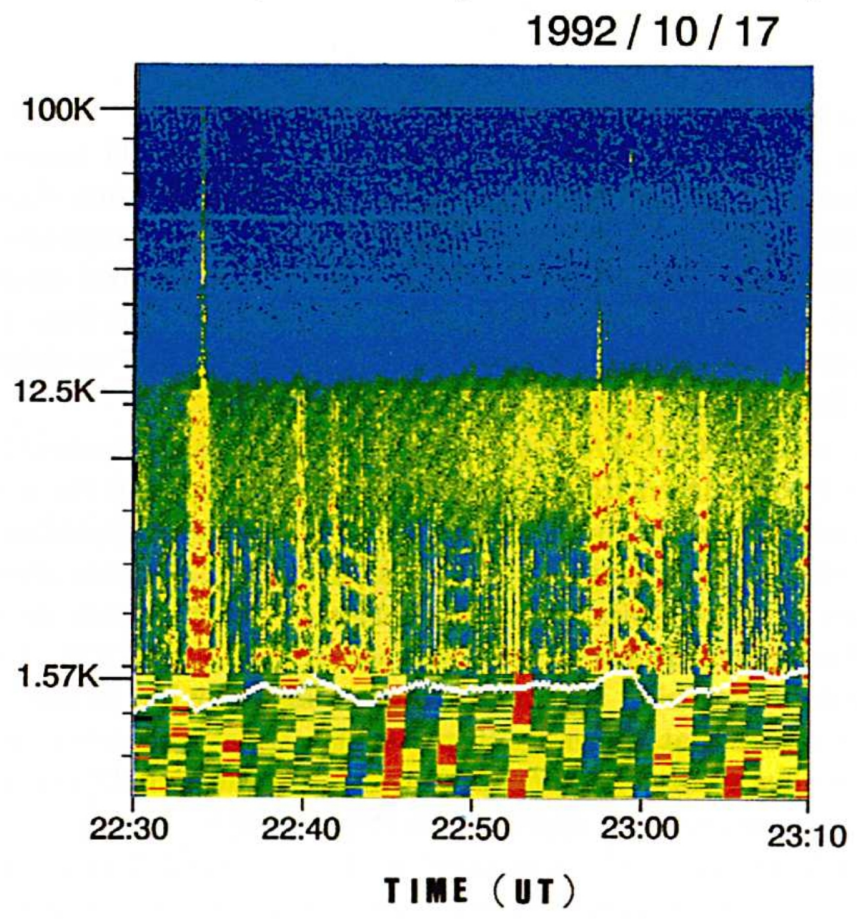

Fig. 27. Magnified frequency spectra of Electron Cyclotron Harmonic (ECH) emissions. These emissions occur together with strong BEN (denoted as the red portion of the BEN spectra). The harmonics of these emissions extend above the electron plasma frequency at $10 \mathrm{kHz}$ as determined from the LCF of CR. 


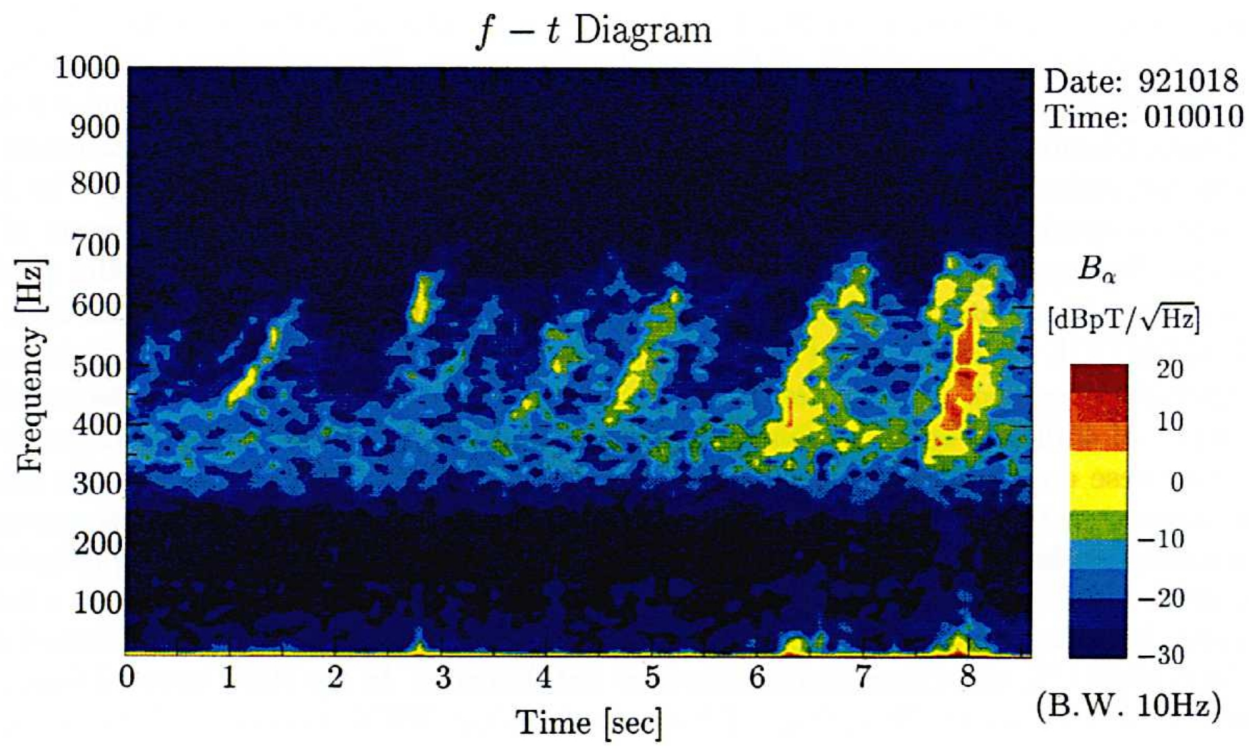

Fig. 28. Time expanded $f-t$ diagram for the magnetic $B_{\alpha}$ component from banded electromagnetic emissions (Chorus) observed at 0100 UT of October 18. This display was generated from the WFC data with an 8.7 sec duration. The characteristic features of Chorus emissions are shown by the rising tones with frequency from 300 to $700 \mathrm{~Hz}$.

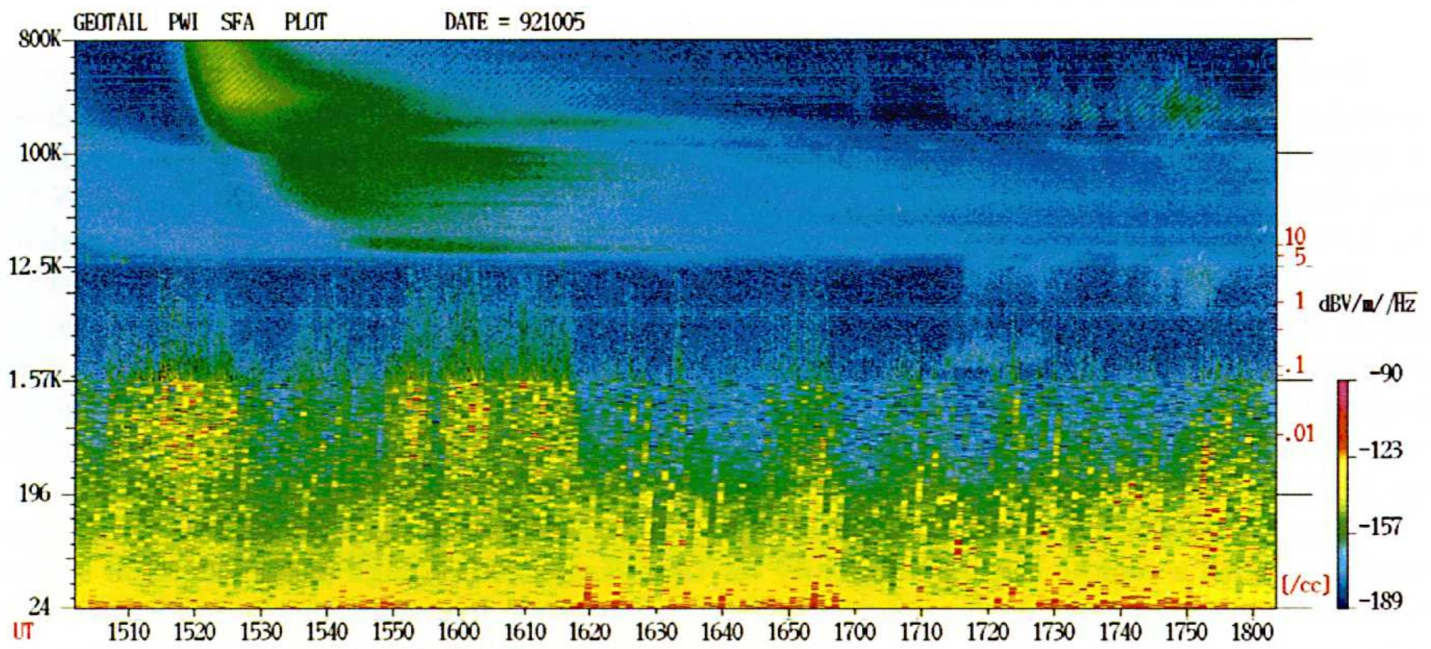

Fig. 29. A typical example of dynamic frequency spectra for solar (or interplanetary) Type III radio bursts observed when the GEOTAIL was out of the magnetosphere. The lower cutoff of the Type III bursts extends to very low frequency with a small dispersion. 
first interval, we can infer that the GEOTAIL traversed regions of outer magnetosphere. During this interval, two significant wave phenomena have been observed. One is strong electromagnetic emissions with a banded structure having a center frequency around 200 to $500 \mathrm{~Hz}$. The bandwidth of the emissions is about $\pm 50 \%$ of their center frequency. The emissions continue to rise in frequency during the entire second interval, and their intensity becomes stronger and stronger. The second wave phenomena observed during this interval is the appearance of the Electron Cyclotron Harmonic emissions (ECH) at frequencies above the tenth higher harmonic. The ECH emissions seem to overlap with strong BEN. Figure 27 shows the magnified $f$ - $t$ diagram of the ECH emissions. Interestingly enough the harmonics overlapping with BEN exceed the electron plasma frequency (as indicated by the LCF of CR) of $8 \mathrm{kHz}$. In the third interval from $2300 \mathrm{UT}$ to $0500 \mathrm{UT}$, GEOTAIL appeared to be in the inner magnetosphere judging from the stable magnetic field intensity observed. We can see very intensive banded electromagnetic emissions with a center frequency of $450 \mathrm{~Hz}$. Figure 28 shows the time expanded $f-t$ diagram of the magnetic $B_{\alpha}$ component for these emissions. This display was generated from the WFC data for the sake of showing the finer structure of the spectrum. From this display, the banded emissions appear to be Chorus emissions which exhibit a rising tone, as has often been observed previously (Tsurutani and Smith, 1974, 1977). The WFC data also shows another type of Chorus emission with a falling tone and hooks, but they are not included herein. Detection of these chorus emissions lasted until 0500 UT. After 0500 UT, the Chorus emissions were not detected. In the third interval from 0500 UT to 0800 UT of October 18, Broadband Electrostatic Noise (BEN) represented the dominant emissions which were detected.

In the third period from 0800 UT to 1415 UT of October 18, the intensities of the ambient magnetic field become a little weaker and it begins to fluctuate. The spectral features suggest that the GEOTAIL spacecraft traversed through or near to the plasmasheet. Further, the background electron density is about $1 / \mathrm{cc}$ and we can see BEN and Electron Cyclotron emissions although their intensities are weak.

\section{Discussion and Conclusions}

To date, the wave receivers for the GEOTAIL-PWI have been functioning properly after the extension of the antennas and magnetic sensors. Though the sensors are affected to a small degree by noise due to photo-electron emission from the surface of the antennas and the spacecraft, the data show a fairly good quality. The data acquisition at UDSC of ISAS and the DSN of NASA has been operating as expected and the subsequent data analysis capabilities are in good shape.

The waves surveyed in the previous section are typically observed in the deep magnetotail and in the dayside magnetosphere/magnetopause. The AKR is frequently observed in the tail and may provide insight into the injection of particles into the near Earth magnetosphere. Also frequently observed is an intensified Auroral Myriametric Radiation (AMR) in the frequency range between $10 \mathrm{kHz}$ and $100 \mathrm{kHz}$ and associated with intense AKR. The interrelation between the AKR and the AMR peaks our interest and will be studied statistically in the future.

Occasionally the solar (or interplanetary) Type III radio bursts are measured by the SFA and provide us with a remote sensing capability for the information on high energy electron streams in the solar wind. A typical example of the observed dynamic frequency spectrum for a Type III radio burst is shown in Fig. 29. The example shows a case when the Type III burst extends to very low frequency with a small dispersion (e.g., Reiner et al., 1992a, 1992b).

The fine frequency resolution of the SFA provides an opportunity to evaluate the fine structure of the space/time variation for the LCF of CR, or equivalently, the local electron density variation. The EFD team monitors a variation in the plasma electric potential relative to the spacecraft potential by using a single element of the PANT. The monitored potential is a measure of the variation in the product of the local electron density and the square root of the local elec- 
tron temperature. Therefore we can estimate the variation in the local electron temperature by comparing the variation in the LCF of CR with the potential variation of the single probe. This will provide a unique opportunity for the study of the temperature profile in the magnetotail.

The BEN are the most intense and most frequently detected electrostatic waves in the magnetotail as first reported by Gurnett et al. (1976) from IMP-8 observations. The GEOTAIL-PWI measurement of BEN will provide additional information to that already obtained by previous spacecraft such as IMP-8 (Gurnett et al., 1976), ISEE-3 (Scarf et al., 1984) and ISEE-1 (Catell et al., 1986). Though there exist many theoretical efforts to interpret the mechanism of BEN generation (Huba et al., 1978; Grabbe, 1987; Schriver and Ashour-Abdalla, 1987), the BEN characteristics appear too complicated to be explained by these theories. Within this context, the GEOTAIL-PWI will provide a unique set of data on the BEN wave forms which can be used to gain further insight into the generation mechanism. The wave forms of BEN have been successfully captured by the WFC receiver which provides information on the phase and amplitude of two electric and three magnetic components simultaneously for a period of $8.8 \mathrm{sec}$ in its Memory mode of operation.

In contrast to the BEN, the MNB are much less frequently observed on GEOTAIL. Though the MNB are typically observed when the spacecraft enters the magnetosheath region (as shown in the example discussed above), they sometimes are observed even in the central plasmasheet co-existing with the BEN. Statistical studies of the association and coupling between BEN and MNB along with correlation studies of geomagnetic indices and the AKR activity would be very beneficial for providing better understanding of the role of the plasma waves in the magnetotail region.

In addition, combining with the magnetic and electric field measurement by the MGF and EFD team, the PWI (especially, the onset and quenching of each wave event) can provide useful information on the global structure of the geomagnetic field such as the plasmoid passage, travelling compression region (TCR) and flapping motion of the tail boundaries. The correlation studies with energetic particle fluxes will be of great interest and provide a stimulus to the development of theories and computer experiments on linear and nonlinear wave-particle interactions.

The PWI project on board the GEOTAIL spacecraft was started under collaboration with wave investigators in the US led by the late F. L. Scarf. He gave us many important comments on wave observations with spacecraft and on the design of the PWI system. To some degree we owe the good quality of data obtained with PWI to his valuable comments.

The PWI team would like to express its sincere thanks to ISAS and NASA for the successful launching of the GEOTAIL spacecraft. We thank the patient efforts by the management team headed by Prof. A. Nishida and Prof. T. Uesugi, without which the GEOTAIL mission would have not been successful. Profs. T. Mukai and T. Yamamoto of ISAS and Messrs. M. Kamimura, M. Kawaguchi and other staff of the NEC Corporation are especially appreciated for their unselfish and enthusiastic assistance and cooperation during the EMC pre-launch testing. We appreciate to Prof. S. Kokubun providing MGF data to calculate the electron cyclotron frequency. The PWI hardware was manufactured by Meisei Electric Company. We owe our thanks to Messrs. Y. Sato and W. Taguchi for their hard work and help which has been shared with some of the co-authors. Assistance by Dr. S. Yagitani, Mr. Y. Usui and graduate students at Kyoto University and Kanazawa University is greatly appreciated. Without their contribution, the data analyses, including the software development for the data acquisition, would not have been possible. We would like to note especially contributions by Messrs. T. Murata, Y. Takeshita, O. Nishimura, T. Miyatake, T. Fujita, and A. Chikuba at Kyoto University and H. Murata and T. Shiozaki at Kanazawa University. We thank Dr. G. K. Crawford for his careful reading of the manuscript. 


\section{REFERENCES}

Anderson, R. R., Plasma waves at and near the neutral sheet, in Proceedings of the Conference on Achievements of the IMS, Eur. Space Agency Spec. Publ., ESA SP-217, 199, 1984.

Anderson, R. R., C. C. Harvey, M. M. Hoppe, B. T. Tsurutani, T. E. Eastman, and J. Etcheto, Plasma waves near the magnetopause, J. Geophys. Res., 87, 2087, 1982.

Cattell, C. A., F. S. Mozer, E. W. Hones, Jr., R. R. Anderson, and R. D. Sharp, ISEE observations of the plasma sheet boundary, plasma sheet, and neutral sheet, 2, Waves, J. Geophys. Res., 91, 5681, 1986.

Coroniti, F. V., F. L. Scarf, C. F. Kennel, and D. A. Gurnett, Continuum radiation and electron plasma oscillations in the distant geomagnetic tail, Geophys. Res. Lett., 11, 661, 1984.

Frandsen, A. M. A., R. E. Holzer, and E. J. Smith, OGO search coil magnetometer experiment, IEEE Trans. Geosci. Electr., GE-6, 61, 1969.

Grabbe, C. L., Numerical study of the spectrum of broadband electrostatic noise in the magnetotail, J. Geophys. Res., 92, 1185, 1987.

Gurnett, D. A., The earth as a radio source: Terrestrial kilometric radiation, J. Geophys. Res., 79, $4227,1974$.

Gurnett, D. A. and L. A. Frank, A region of intense wave turbulence on auroral field lines, J. Geophys. Res., 82, $1031,1977$.

Gurnett, D. A. and L. A. Frank, Plasma waves in the polar cusp: Observations from Hawkeye 1, J. Geophys. Res., 82, 1031, 1978.

Gurnett, D. A., L. A. Frank, and R. P. Lepping, Plasma waves in the distant magnetotail, J. Geophys. Res., 81, $6059,1976$.

Gurnett, D. A., F. L. Scarf, R. W. Fredericks, and J. E. Smith, The ISEE-1 and ISEE-2 plasma wave investigation, IEEE Trans. Geosci. Electr., GE-16, 225, 1978.

Gurnett, D. A., R. R. Anderson, B. T. Tsurutani, E. J. Smith, G. Paschmann, G. Haerendel, S. J. Bame, and C. T. Russell, Plasma wave turbulence at the magnetopause: Observations from ISEE 1 and 2, J. Geophys. Res., 84, 7043, 1979.

Gurnett, D. A., W. Calvert, and R. L. Huff, The polarization of escaping terrestrial continuum radiation, J. Geophys. Res., 93, 12817, 1988.

Hashimoto, K., Tail dynamics and plasma waves, Science Objectives of GEOTAIL Mission, Proc. Magnetosphere Ionosphere Symposium, 31, 1985.

Huba, J. D., N. T. Gladd, and K. Papadopoulos, Lower-hybrid-drift wave turbulence in the distant magnetotail, J. Geophys. Res., 83, 5217, 1978.

Kaiser, M. L. and J. K. Alexander, Relationship between auroral substorms and the occurrence of terrestrial kilometric radiation, J. Geophys. Res., 82, 5283, 1977.

Kennel, C. F., F. V. Coroniti, and F. L. Scarf, Plasma waves in Magnetotail flux ropes, J. Geophys. Res., 91, $1424,1986$.

Kokubun, S., T. Yamamoto, M. H. Acuna, K. Hayashi, K. Shiokawa, and H. Kawano, The GEOTAIL magnetic field experiment, J. Geomag. Geoelectr., this issue, 7-21, 1994.

Lee, L. C., J. R. Kan, and C. S. Wu, Generation of auroral kilometric radiation region, Planet. Space Sci., 28, $703,1980$.

Matsumoto, H. and Plasma Wave Team, Plasma wave observation on GEOTAIL, Science Objectives of GEOTAIL Mission, Proc. Magnetosphere Ionosphere Symposium, 29, 1985.

Nagano, I., Plasma waves in the tail and boundary layer, Science Objectives of GEOTAIL Mission, Proc. Magnetosphere Ionosphere Symposium, 35, 1985.

Nishida, A., T. Hada, K. A. Anderson, R. R. Anderson, S. J. Bame, and E. W. Hones, Broadband electrostatic noise in the magnetotail: Its relation to plasma sheet dynamics, J. Geophys. Res., 90, 4453, 1985.

Omura, Y., Theory and simulation studies of the plasma turbulence in the magnetospheric tail, Science Objectives of GEOTAIL Mission, Proc. Magnetosphere Ionosphere Symposium, 33, 1985.

Reiner, M. J., R. G. Stone, and J. Fainberg, Unusual radio and plasma wave phenomena observed in March 1991, Geophys. Res. Lett., 19, 1271, 1992a.

Reiner, M. J., R. G. Stone, and J. Fainberg, Observation of non-drifting radio emissions associated with the intense solar activity in March 1991, Geophys. Res. Lett., 19, 1275, 1992b.

Rodriguez, P. and D. A. Gurnett, Electrostatic and electromagnetic turbulence associated with the earth's bow shock, J. Geophys. Res., 80, 19, 1975.

Scarf, F. L., ISEE-3 plasma wave observations in the earth's geomagnetic tail, Science Objectives of GEOTAIL Mission, Proc. Magnetosphere Ionosphere Symposium, 27, 1985.

Scarf, F. L., R. W. Fredericks, I. M. Green, and C. T. Russell, Plasma waves in the daytime polar cusp, 1, Magnetospheric observations, J. Geophys. Res., 77, 2274, 1972.

Scarf, F. L., L. A. Frank, K. L. Ackerson, and R. P. Lepping, Plasma wave turbulence at distant crossings of plasma sheet boundaries and the neutral sheet, Geophys. Res. Lett., 1, 189, 1974.

Scarf, F. L., R. W. Fredericks, D. A. Gurnett, and E. J. Smith, The ISEE-C plasma wave investigation, IEEE Trans. Geosci. Electr., GE-16, 191, 1978.

Scarf, F. L., J. K. Alexander, R. A. Helliwell, M. Kelly, P. Kellogg, P. M. Kintner, W. S. Kurth, H. Matsumoto, 
P. Rodriguez, S. D. Shawhan, E. J. Smith, R. G. Stone, and W. W. L. Taylor, A plasma wave investigation for the OPEN/EML spacecraft, TRW Proposal, No. 36749, 1980.

Scarf, F. L., F. V. Coroniti, C. F. Kennel, R. W. Fredericks, D. A. Gurnett, and E. J. Smith, ISEE-3 wave measurements in the distant geomagnetic tail and boundary layer, Geophys. Res. Lett., 11, 335, 1984a.

Scarf, F. L., F. V. Coroniti, C. F. Kennel, E. J. Smith, J. A. Slavin, B. T. Tsurutani, S. J. Bame, and W. C. Feldman, Plasma wave spectra near slow mode shocks in the distant magnetotail, Geophys. Res. Lett., 11, 1050, 1984b.

Schriver, D. and M. Ashour-Abdalla, Generation of high-frequency broadband electrostatic noise: The role of cold electrons, J. Geophys. Res., 92, 5807, 1987.

Schriver, D. and M. Ashour-Abdalla, Broadband electrostatic noise due to field aligned currents, Geophys. Res. Lett., 16, 889, 1989.

Schriver, D. and M. Ashour-Abdalla, Cold plasma heating in the plasma sheet boundary layer: Theory and simulations, J. Geophys. Res., 95, 3987, 1990.

Sibeck, D. G., G. L. Siscoe, J. A. Slavin, E. J. Smith, S. J. Bame, and F. L. Scarf, Magnetotail flux ropes, Geophys. Res. Lett., 11, 1090, 1984.

Slavin, J. A., E. J. Smith, B. T. Tsurutani, D. G. Sibeck, H. J. Singer, D. N. Baker, J. T. Gosling, E. W. Hones, and F. L. Scarf, Substorm associated traveling compression regions in the distant tail: ISEE-3 geotail observations, Geophys. Res. Lett., 11, 657, 1984.

Tsuduki, Y., M. Nakamura, K. Tsuruda, H. Hayakawa, A. Matsuoka, and T. Okada, An Analysis of Single Probe Data on GEOTAIL, Proc. 1993 Japan Earth and Planetary Science Joint Meeting, H22-11, 1993.

Tsuruda, K., H. Hayakawa, M. Nakamura, T. Okada, A. Matsuoka, F. S. Mozer, and R. Schmidt, Electric field measurements on the GEOTAIL satellite, J. Geomag. Geoelectr., 1994 (submitted).

Tsurutani, B. T. and E. J. Smith, Postmidnight chorus: A substorm phenomenon, J. Geophys. Res., 79, 118, 1974.

Tsurutani, B. T. and E. J. Smith, Two types of magnetospheric ELF chorus and their substorm dependencies, $J$. Geophys. Res., 82, 5112, 1977.

Tsurutani, B. T. and T. T. von Rosenvinge, ISEE-3 distant GEOTAIL results, Geophys. Res. Lett., 11, 1027, 1984.

Tsutsui, M., R. J. Strangeway, B. T. Tsurutani, H. Matsumoto, J. L. Phillips, and M. Ashour-Abdalla, Wave mode identification of electrostatic noise observed with ISEE3 in the deep tail boundary layer, J. Geophys. Res., 96, 14065, 1991.

Tsutsui, M., H. Kojima, I. Nagano, H. Sato, T. Okada, H. Matsumoto, T. Mukai, and M. Kawaguchi, Magnetic radiations from harness wires of spacecraft, IEICE Trans. Commun., E75-B, 174, 1992.

Ungstrup, E., A. Bahnsen, H. K. Wong, M. Andre, and L. Matson, Energy source and generation mechanism for auroral kilometric radiation, J. Geophys. Res., 95, 5973, 1990.

Voots, G. R., D. A. Gurnett, and S.-I. Akasofu, Auroral kilometric radiation as an indicator of auroral disturbances, J. Geophys. Res., 82, 2259, 1977.

Wu, C. S. and L. C. Lee, A theory of the terrestrial kilometric radiation, Astrophys. J., 230, $621,1979$.

Wu, C. S., H. K. Wong, D. J. Gorney, and L. C. Lee, Generation of the auroral kilometric radiation, J. Geophys. Res., 87, 4476, 1982. 